\title{
Impacts of Increasing Low-Level Shear on Supercells during the Early Evening Transition*
}

\author{
Brice E. COFFER AND MATTHEw D. PARKer \\ North Carolina State University, Raleigh, North Carolina
}

(Manuscript received 15 October 2014, in final form 13 January 2015)

\begin{abstract}
The dynamical response of simulated supercells to temporally increasing lower-tropospheric vertical wind shear is investigated using idealized simulations. These simulations are based upon observed soundings from two cases that underwent an early evening transition during the Second Verification of the Origins of Rotation in Tornadoes Experiment (VORTEX2). Mature supercells were simulated in observed afternoon environments with moderate vertical wind shear and then compared to simulated supercells experiencing observed evening increases in lower-tropospheric shear. The primary effect of the increase in low-level shear is to establish larger values of vertical vorticity at lower altitudes in the storm's updraft. In turn, this leads to a nonlinear increase in the updraft strength due to the enhanced dynamic pressure minimum associated with larger vorticity in the storm's mesocyclone. This is particularly important at low levels, where it increases the storm's ability to lift cool surface air (including outflow). Trajectories launched in developing vortices show that, despite comparable buoyant accelerations, parcels experience greater vertical velocity and stretching of vertical vorticity due to increased dynamic accelerations when the low-level shear is increased. Thus, even as low-level stability gradually increases in the early evening, the supercells' low-level updraft intensity and surface vorticity production can increase. These results are consistent with climatological observations of a supercell's likelihood of tornadogenesis during the early evening hours.
\end{abstract}

\section{Introduction}

Supercell mesocyclonic tornadogenesis has been described as a three-step process (Davies-Jones and Brooks 1993; Davies-Jones 2015). First, a thunderstorm acquires rotation through tilting of environmental horizontal vorticity into the vertical. Second, generation of horizontal vorticity and tilting via a downdraft produces vertical vorticity at the surface. Finally, a tornadic circulation spins up at the ground as vertical vorticity is converged and stretched into a deep, vertically oriented vortex. This final step is still not easily anticipated, as several factors, including outflow buoyancy and the superposition of the surface circulation with strong lifting, determine whether

\footnotetext{
* Supplemental information related to this paper is available at the Journals Online website: http://dx.doi.org/10.1175/MWR-D14-00328.s1.

Corresponding author address: Brice E. Coffer, Department of Marine, Earth, and Atmospheric Sciences, North Carolina State University, Campus Box 8208, Raleigh, NC 27695-8208.

E-mail: becoffer@ncsu.edu
}

surface parcels with cyclonic vorticity will ascend to appreciable heights (Markowski et al. 2011; Markowski and Richardson 2014). Although most significant tornadoes are associated with supercells, most supercells do not produce tornadoes (e.g., Trapp et al. 2005). Thus, one of the biggest remaining challenges in supercell research is discriminating between tornadic and nontornadic supercells. Several environmental parameters have been shown to possess substantial skill in forecasting tornadogenesis. One such parameter is the environmental shear vector magnitude ${ }^{1}$ in the lowest kilometer above ground level (AGL; Brooks et al. 2003; Markowski et al. 2003). Trying to understand the physical importance of low-level shear was a primary motivation for the present study.

It has been shown through numerical and observational studies that parcels reaching the near-surface ${ }^{2}$ vortex have a history of horizontal vorticity generation and tilting during descent through a downdraft (e.g.,

\footnotetext{
${ }^{1}$ The lower-tropospheric environmental vertical wind shear vector magnitude is hereafter referred to as simply low-level shear.

${ }^{2}$ In this study, near surface will refer to the lowest model levels (below $100 \mathrm{~m}$ ), while low level will refer to lowest 1-2 km AGL.
} 
Davies-Jones and Brooks 1993; Adlerman et al. 1999; Markowski et al. 2008, 2012a,b; Dahl et al. 2014). If the surface vertical vorticity that develops into tornadoes is produced solely due to downdraft processes, then why is the low-level shear magnitude of the inflow a statistically significant predictor of strong tornadoes? One possibility is that the production of vertical vorticity by downdrafts is sensitive to the low-level storm-relative wind [e.g., as suggested by Davies-Jones et al. (2001)], which in turn is linked to the structure of the environmental hodograph. In addition, tornadic vortices appear to form when vorticity-rich outflow can be readily ingested by the overlying updraft (Markowski and Richardson 2014). This is most likely to occur when the cold pool has weak negative buoyancy, when a strong upward perturbation pressure gradient force exists, and when the surface circulation remains in an area of strong convergence underneath the low-level and midlevel mesocyclone (Markowski et al. 2002; French et al. 2008; Marquis et al. 2012; Markowski and Richardson 2014). In contrast, within three nontornadic supercells, Markowski et al. (2011) found that trajectories in the outflow rose only a few hundred meters before abruptly decelerating. They hypothesized that, in such cases, the perturbation pressure-gradient force was insufficiently strong (or even adverse), preventing parcels from being converged and stretched into a tornadic vortex. The amount of shear concentrated in the near-ground layer might be critically important for tornadic supercells because it governs the base height of the midlevel mesocyclone (Markowski et al. 2012b), which is also associated with a local minimum in the dynamic pressure field. A stronger mesocyclone at a lower altitude would allow the storm to forcibly lift and stretch negatively buoyant air from the rear-flank outflow through an upward-directed perturbation pressure-gradient force.

The role of low-level environmental shear was one of many questions motivating the Second Verification of the Origins of Rotation in Tornadoes Experiments (VORTEX2; Wurman et al. 2012), which collected unprecedented observations of numerous supercells. One mission of this campaign was to understand spatial and temporal variability in the environment of supercells through numerous near-storm soundings (Parker 2014). When comparing early-in-life versus late-in-life near-supercell soundings, Parker (2014) found that, although the 0-6-km shear vector magnitude remained nearly identical over time, the winds below 2-3 km AGL increased in speed near sunset. This resulted in dramatic increases in low-level environmental bulk shear and storm-relative helicity (SRH) as supercells matured late in the day.

This early evening increase in low-level shear is a commonly observed characteristic of severe weather events in the central United States (Maddox 1993), and the U.S. tornado climatology shows a maximum frequency of tornadoes during this time frame, ${ }^{3}$ especially in the Great Plains. Not coincidentally, the Great Plains also exhibits a distinct maximum in the climatology of nocturnal low-level jets (LLJ; Bonner 1968; Song et al. 2005). Decoupling of the surface layer from the rest of the afternoon boundary layer during the early evening transition (EET) commonly causes a decrease in the surface wind speed (Nieuwstadt 1985; Lapworth 2003) and the development of an LLJ (e.g., Blackadar 1957; Shapiro and Fedorovich 2010; Van de Wiel et al. 2010), the combination of which increases the lowertropospheric vertical wind shear. A defining characteristic of the LLJ is a veering of the wind vector with time and the development of a supergeostrophic wind maximum (Stull 1988). These temporal changes in the magnitude and direction of the lower-tropospheric wind shear are, in turn, potentially important to convective storm dynamics.

For some time storm researchers have hypothesized that the early evening increase in lower-tropospheric shear could have profound impacts on convective storm dynamics, including MCSs (e.g., Fritsch et al. 1994), as well as supercells and their likelihood of tornado production (e.g., Maddox 1993; Markowski et al. 1998b). Mead and Thompson (2011) found that SRH can increase by $100-200 \mathrm{~m}^{2} \mathrm{~s}^{-2}$ from 0000 to 0300 UTC (i.e., during sunset in the central United States) for significant tornado events, and although not a part of the formal literature, forecast discussions issued by the National Oceanic and Atmospheric Administration (NOAA) Storm Prediction Center (SPC) frequently mention a time window for increased probability of tornadogenesis during the EET due to LLJ-enlarged hodographs. This window presumably closes once significant surface cooling occurs and updrafts are no longer surface based, although Nowotarski et al. (2011) showed that simulated supercells could overcome rather substantial stabilization due to dynamic lifting.

In addition to questions about how storms respond to changes in low-level shear during the EET, it is also fundamentally unclear how changes in shear interplay with changes in stability within typical supercellular

\footnotetext{
${ }^{3}$ These data are available from http://www.ncdc.noaa.gov/ climate-information/extreme-events/us-tornado-climatology/trends. Storm chasers often anecdotally refer to this phenomenon as "sixo'clock magic," because supercells seem to have the propensity to produce tornadoes in the early evening hours, as the sun sets. This may be associated with lower lifted condensation levels (LCL) in addition to the higher SRH (e.g., Parker 2014), as well as the cessation of boundary layer convection (e.g., Nowotarski et al. 2015).
} 
environments. The EET has been predominately studied with cases that exhibit clear skies and light winds [in order to distinctly isolate the signatures of the EET; Acevedo and Fitzjarrald (2001)]. Soundings from near supercells (e.g., Parker 2014) exhibit stronger wind speeds and shear through the depth of the afternoon boundary layer than what is typically documented in the EET climatology studies. The onset of the EET also could be accelerated in the inflow sector of many supercells; for the common situation where the inflow sector is generally east of the supercell's cloud mass, late afternoon shading of solar radiation may trigger localized surface cooling (Markowski et al. 1998a). Numerical modeling studies that account for this radiative cooling beneath supercell anvil clouds reveal reductions in vertical mixing and the development of stronger vertical shear below $500 \mathrm{~m}$ AGL (Frame and Markowski 2010, 2013).

The focus of the present study is on the response of supercells' low-level updrafts to increasing low-level shear during the EET (without changes to the thermodynamic profile), and the associated evolution and amplification of near-surface vertical vorticity. We hypothesized that the evolving low-level shear during the EET leads to changes in the storm's profile of vertical vorticity that subsequently enhance the lowlevel dynamic lifting of near-surface air. To address this hypothesis, we employ idealized simulations based upon observed soundings from nearby VORTEX2 supercells that underwent EETs. Details regarding the methods are described in section 2. Results and interpretation from the two VORTEX2 cases are offered in sections 3 and 4 . A summary of the main conclusions and avenues for future work are presented in section 5 .

\section{Methods}

\section{a. Model configuration}

We performed idealized supercell simulations using version 17 of the Bryan Cloud Model 1 [CM1; Bryan and Fritsch (2002); CM1 is available online at www2. $\mathrm{mmm}$.ucar.edu/people/bryan/cm1/], which is a nonhydrostatic cloud-scale model. Storms were simulated for $5 \mathrm{~h}$ on a $150 \mathrm{~km} \times 150 \mathrm{~km} \times 18 \mathrm{~km}$ domain having horizontal grid spacing of $250 \mathrm{~m}$ and vertical grid spacing that was stretched from $50 \mathrm{~m}$ near the surface to $250 \mathrm{~m}$ aloft. The horizontally homogeneous simulations were initialized with slightly modified observed soundings, based on two VORTEX2 cases that will be discussed further in section $2 \mathrm{c}$. Convection was triggered using the convergence mechanism described by Loftus et al. (2008). For simplicity and continuity with other recent work, we employed the microphysical parameterization developed by Lin et al. (1983), as modified by the National Aeronautics and Space Administration (NASA) Goddard Cumulus Ensemble model (Tao and Simpson 1993). The general conclusions presented herein were insensitive to several microphysical parameterization sensitivity tests, including the dual-moment Thompson (Thompson et al. 2008) and Morrison (Morrison et al. 2005) schemes, as well as the National Severe Storms Laboratory's variable graupel and hail density scheme (Mansell et al. 2010). Open, radiative lateral boundary conditions were employed (e.g., Klemp and Wilhelmson 1978), with rigid, free-slip ${ }^{4}$ boundary conditions on the lower and upper boundaries, and a Rayleigh damping sponge layer was applied above $14 \mathrm{~km}$. Radiation, surface fluxes, and the Coriolis force were neglected in the interest of isolating only a few key processes. Rather than attempting to reproduce an EET using the model's physical parameterizations, the EET was instead introduced by incorporation of observed VORTEX2 soundings, as is explained next.

\section{b. Base-state substitution}

The storm-scale modeling community has relied heavily on homogeneous base-state conditions in order to isolate and understand fundamental dynamical processes in convective storms [as reviewed comprehensively by Wilhelmson and Wicker (2001)]. Simulating convection in heterogeneous environments introduces several complicating factors to both experimental design and the interpretation of cause versus effect. To address such concerns in this study, we employ an approach that emulates temporal tendencies experienced by a storm by gradually changing the homogeneous model environment [much as developed by Richardson et al. (2007) and the references therein, as well as Letkewicz et al. (2013)]. We adopt the specific formulation of Letkewicz et al. (2013), which they termed base-state substitution (BSS). This allows the user to "investigate how a mature storm responds to a changing background environment, as opposed to how storms develop in different background environments" (Letkewicz et al. 2013, p. 3063). The BSS approach provides an ideal platform to test how a mature supercell responds to the EET. Once

\footnotetext{
${ }^{4}$ In one recent study (Schenkman et al. 2014), frictionally generated vorticity was found to be a large contributor to supercell tornadogenesis in a simulated case study using data-assimilation. However, there is not yet widespread agreement on how best to apply surface drag in idealized simulations of sheared environments, so this is a subject for future research.
} 
TABLE 1. Summary of common environmental forecasting parameters calculated from the observed VORTEX2 soundings for (top) 5 Jun 2009 “Goshen County" tornadic supercell, (middle) 18 May 2010 "Dumas, Texas," tornadic supercell, and (bottom) 12 May 2010 "Clinton, Oklahoma," Tornadic Supercell. Environmental bulk layer shear vector magnitudes are referred to as bulk wind difference $\left(\mathrm{BWD} ; \mathrm{m} \mathrm{s}^{-1}\right)$. Storm-relative helicity $\left(\mathrm{SRH} ; \mathrm{m}^{2} \mathrm{~s}^{-2}\right.$ ) was calculated using the Bunkers storm motion (Bunkers et al. 2000). Both the convective available potential energy (CAPE; $\mathrm{J} \mathrm{kg}^{-1}$ ) and lifted condensation level (LCL; $\mathrm{m}$ ) use the surface-based parcel, and the supercell composite parameter (SCP) and significant tornado parameter (STP) (dimensionless) utilize the effective inflow layer described by Thompson et al. (2007). The details of the Clinton supercell simulation are not presented herein because of reasons discussed in section $5 \mathrm{~b}$.

\begin{tabular}{|c|c|c|c|c|c|c|c|c|}
\hline & 0-6-km BWD & 0-1-km BWD & 0-1-km SRH & 0-3-km SRH & CAPE & LCL & SCP & STP \\
\hline \multicolumn{9}{|l|}{ Goshen } \\
\hline CONTROL-2155 UTC & 30 & 6 & 84 & 142 & 2600 & 1130 & 8 & 2 \\
\hline BSS1-2335 UTC & 31 & 7 & 115 & 219 & 2600 & 1130 & 13 & 4 \\
\hline BSS2—0057 UTC & 30 & 12 & 279 & 381 & 2600 & 1130 & 29 & 8 \\
\hline \multicolumn{9}{|l|}{ Dumas } \\
\hline CONTROL-2257 UTC & 21 & 7 & 90 & 159 & 2132 & 880 & 8 & 2 \\
\hline BSS1-0044 UTC & 23 & 12 & 284 & 429 & 2132 & 880 & 24 & 7 \\
\hline BSS2—0130 UTC & 23 & 13 & 298 & 493 & 2132 & 880 & 27 & 8 \\
\hline \multicolumn{9}{|l|}{ Clinton } \\
\hline CONTROL—0039 UTC & 39 & 15 & 309 & 379 & 2376 & 1560 & 44 & 11 \\
\hline BSS-0139 UTC & 40 & 22 & 444 & 494 & 2376 & 1560 & 59 & 15 \\
\hline
\end{tabular}

a supercell matured in the control simulation, the original base-state wind profile was gradually modified to have stronger low-level shear (as observed), evolving through intermediary observed soundings to the final observed profile. Comparisons will be made between the unmodified, lower-shear control simulation and a highershear BSS simulation.

Unless otherwise stated, in order to isolate the effect of increasing low-level shear on mature supercells, the BSS soundings were thermodynamically identical to the control sounding. The lower-level winds reflected the observed changes in low-level shear, while the upper-level winds in the BSS soundings were unchanged from the control sounding; the breakpoint altitude between the modified and unmodified winds differed between cases [as detailed in sections $2 c(1)$ and $2 c(2)]$ and was based on our subjective assessment of the layer over which the lowertropospheric winds differed the least from time to time in each case. The evolving low-level wind profile alone results in noticeable differences in common environmental forecast indices such as the supercell composite parameter and significant tornado parameter (Thompson et al. 2003), despite identical thermodynamic fields and approximately equal deep layer shear, as shown in Table 1.

For each case, a supercell was simulated in a lowershear environment using the control wind profile in Table 1 and Figs. 1 and 2. In the control run, the wind profile remained unmodified throughout the entire duration of the simulation ( $5 \mathrm{~h}$ ). Using BSS, another simulation was generated starting with the restart file from the control supercell simulation at $t=1.5 \mathrm{~h}$. The BSS process incorporated 10 soundings with increasing shear that were linearly interpolated in time from the three original VORTEX2 observed soundings (Control, BSS1, BSS2 in Table 1 and Figs. 1 and 2); these 10 intermediary soundings were introduced every $6 \mathrm{~min}$ over a span of $1 \mathrm{~h}$. For example, at $t=2 \mathrm{~h}$, the wind profile had evolved to the second VORTEX2 sounding (BSS1), and by $t=2.5 \mathrm{~h}$, the wind profile had completely transitioned to the final VORTEX2 sounding (BSS2). In this way, the original mature supercell experienced a transition to the observed higher-shear environment (from later in the day) in the BSS run.

After the BSS process was completed, the simulation freely evolved for the remaining $2.5 \mathrm{~h}$. Although the 1-h BSS window was somewhat brief (the observed soundings spanned closer to $3 \mathrm{~h}$ ), it provided a longer post-BSS period for comparisons and analysis before convection grew upscale in the control simulations. Comparisons between runs were only performed while both simulations maintained supercell-like structures in the model output reflectivity field (e.g., weak-echo regions, hook echo, etc.). Sensitivity tests were performed varying the timing and duration of the basestate substitution process. Storm evolution was not substantially different among the several simulations, which is consistent with the findings reported by Letkewicz et al. (2013).

\section{c. VORTEX2 case studies}

Rarely have soundings been obtained throughout the life cycle of multiple supercells, especially into the evening transition. The observations from VORTEX2 allow us to test our hypotheses using direct measurements 


\section{June 2009 - Goshen County}

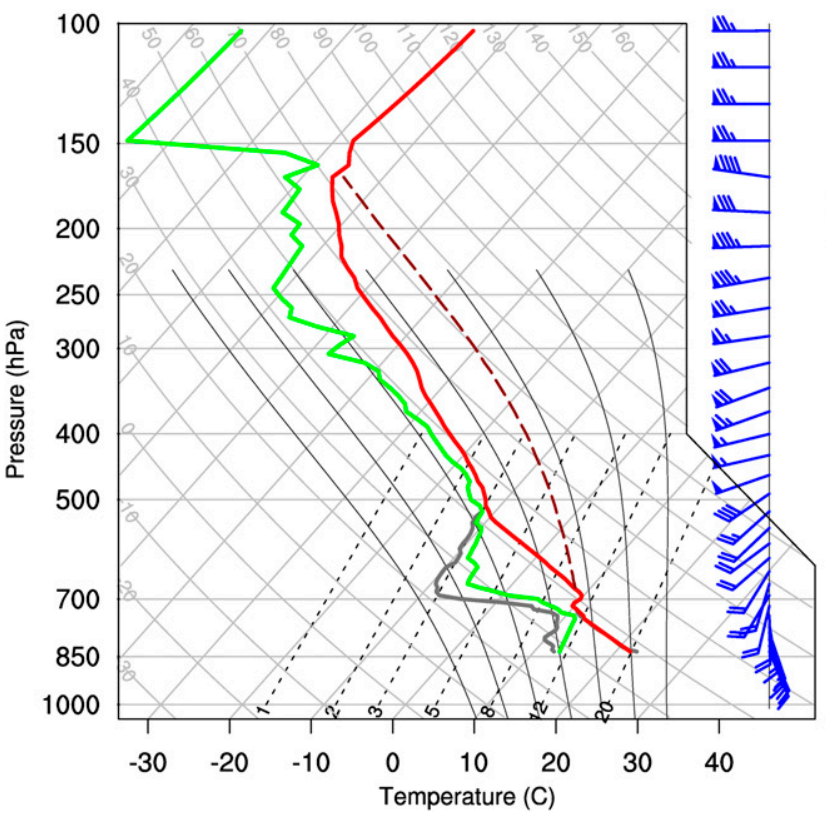

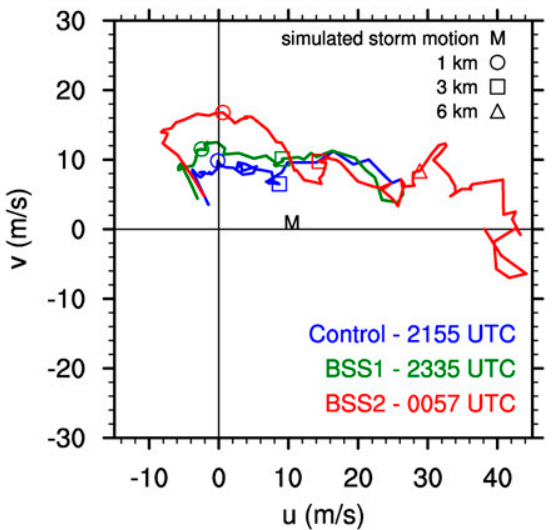

FIG. 1. (left) Skew $T$-log $p$ diagram and (right) hodograph showing the thermodynamic and kinematic environment used in the horizontally homogenous base-state substitution (BSS) simulations for the 5 Jun 2009 "Goshen County" Tornadic Supercell case. The skew $T-\log p$ shows the 2155 UTC Control thermodynamic profile. The sounding displayed is directly from the model's initial conditions and the modifications to the Control sounding are described section 2c. Temperature is in red and dewpoint temperature is in green, while the dashed brown line indicates the path of a surface based parcel above the level of free convection. The wind barbs on the skew $T-\log p \operatorname{correspond}$ to the Control sounding and are displayed in $\mathrm{kt}\left(1 \mathrm{kt}=0.5144 \mathrm{~m} \mathrm{~s}^{-1}\right)$. The unmodified VORTEX2 thermodynamic profile is plotted in dark gray (see section $2 \mathrm{c}$ for more details on the modifications). On the hodographs, the 1-, 3-, and 6-km data points are denoted with symbols as shown, with the simulated storm motion plotted with "M". Control (blue), BSS1 (green), and BSS2 (red) wind profiles refer to the 2155, 2335, and 0057 UTC soundings respectively.

of low-level shear during the EET. Since the supercell simulations in this study are idealized, with horizontally homogeneous initial conditions, the goal of this research is not to reproduce the exact supercell or tornado observed during the cases described below. To make the idealized modeling approach viable, a few subtle modifications to the observed soundings were needed. For example, a stumbling point seems to be that afternoon boundary layers in supercellular environments have both neutral static stability (often even unstable) and strong vertical wind shear. This engenders Kelvin-Helmholtz instability and leads to vigorous mixing. In nature, steep lapse rates and strong shear can persist due to the presence of continual surface heat fluxes, a large-scale pressure gradient, and surface drag. In our idealized model, such energy sources and sinks are absent, and the release of KelvinHelmholtz instability quickly reduces the magnitude of the lower-tropospheric shear. Therefore, we slightly stabilized the temperature profile, which preserves most of the low-level shear without having to otherwise modify the background subgrid-scale eddy mixing tendency in the model's equations. ${ }^{5}$ Other small modifications are described below. ${ }^{6}$

\section{1) 5 JunE 2009: “GOSHEN COUNTY” TORNADIC SUPERCELL}

The Goshen County, Wyoming, tornadic supercell of 5 June 2009 was selected as an ideal case, because of dense temporal and spatial sounding observations through the early evening hours. Three far inflow soundings were chosen to represent the base-state environment during the EET at 2155, 2335, and 0057 UTC (Fig. 1). Only far inflow soundings were considered

\footnotetext{
${ }^{5}$ Experiments were conducted with and without the base-state eddy mixing tendency. This change led to storms that evolved differently. Eliminating the mixing of the base-state environment (i.e., applying mixing only to perturbation fields) has the desirable effect of better preserving large vertical wind shear in the far field environment. However, it may have questionable consequences within the storm itself.

${ }^{6}$ Modified sounding files are available upon request for reproducibility purposes.
} 
18 May 2010 - Dumas, Texas

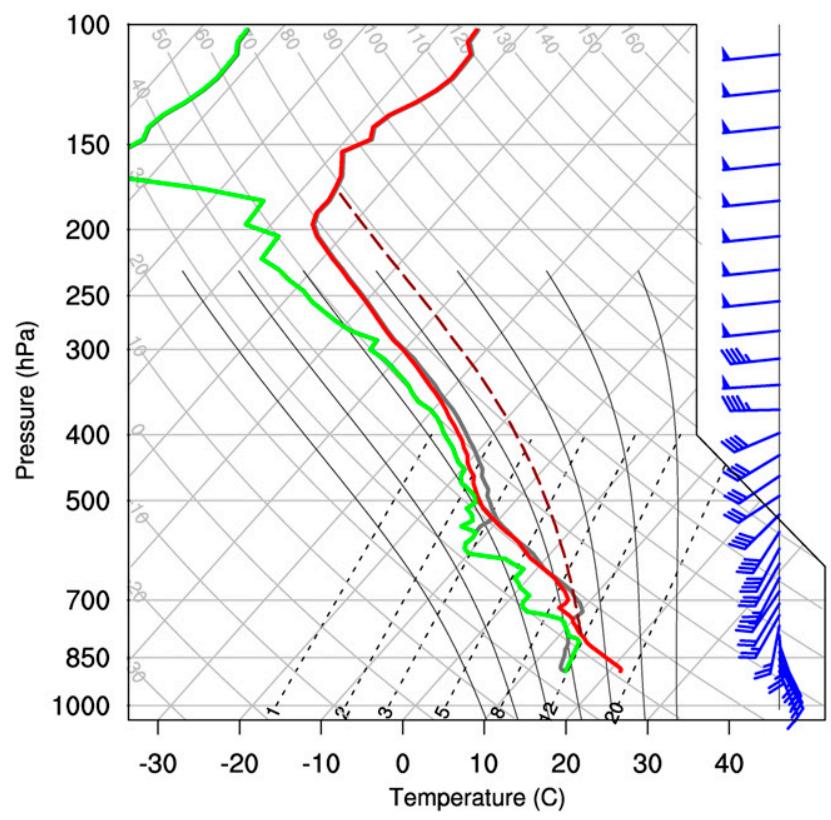

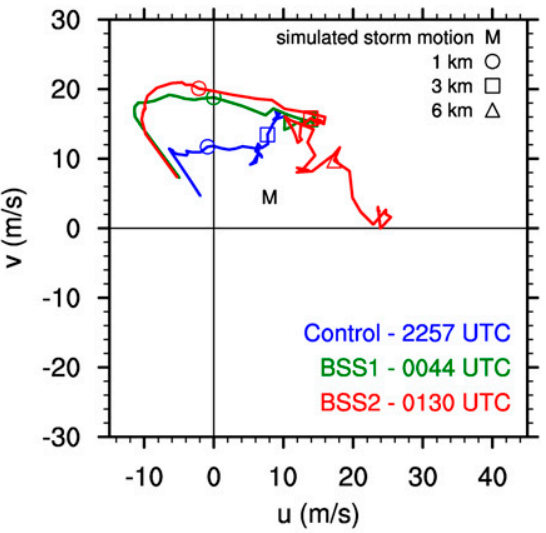

FIG. 2. As in Fig. 1, but for the 18 May 2010 “Dumas, Texas," Tornadic Supercell. Control, BSS1, and BSS2 wind profiles refer to the 2257, 0044, and 0130 UTC soundings, respectively. The observed wind profile transition is rather abrupt because of a data gap due to technical communication issues that plagued the balloon launches between the 2257 and 0044 UTC soundings. because Parker (2014) found substantial differences in LCL height and SRH closer to supercells, presumably due to storm-generated feedbacks. Naturally, the observed storm-following sounding changes also include spatial heterogeneity (in addition to temporal evolution of the environment). For this case, the total distance between the launch points for the three soundings was $80 \mathrm{~km}$, which we consider to be modest given the gently sloping terrain and the absence of a notable airmass boundary.

A few modifications to the 2155 UTC control sounding were necessary. Data collection from soundings in VORTEX2 was normally terminated approximately an hour after launch so that a new sounding could be released. During this hour, the balloons usually reached the approximate height of the tropopause. For CM1 to run properly, stratospheric data needed to be approximated, thus a potential temperature inversion of $20 \mathrm{~K} \mathrm{~km}^{-1}$ with $1 \%$ relative humidity and constant winds were chosen to represent this data gap. These choices above $10 \mathrm{~km}$ likely have little influence on the supercell, especially at low levels. As noted above, the lowest levels were slightly stabilized to prevent spontaneous overturning at the smallest resolvable scales. Potential temperature and mixing ratio were reset to be constant throughout the boundary layer based on their original mean mixed-layer values. Through trial and error, it was also determined that long-lived convection would only occur in the model when the dry, elevated mixed layer was slightly moistened from $2-4 \mathrm{~km}$ (approximately $15 \%$ ). The conclusions presented in this study should be insensitive to this moistening since the thermodynamic profile remained constant throughout the main BSS experiments. Based on the observed wind profiles for this case, the upper bound for the lowertropospheric wind modifications during BSS was chosen to be $6 \mathrm{~km}$ AGL.

\section{2) 18 May 2010: "Dumas, Texas,” TORnadic SUPERCELL}

Simulations were also performed using soundings collected during the Dumas, Texas, supercell of 18 May 2010 from VORTEX2. Three far inflow soundings characterized the EET at 2257, 0044, and 0130 UTC (Fig. 2). The control 2257 UTC sounding was modified in a similar manner to the Goshen case. Originally, the presence of a strong capping inversion near $720 \mathrm{hPa}$ appeared to prevent long-lived convection in the model. Therefore, we modified the sounding by lifting the layer at the base of the inversion by $200 \mathrm{~m}$ (Fig. 2), such as might occur in response to gentle synoptic lifting (e.g., Markowski and Richardson 2010, see their Fig. 7.9). We 
found that the lack of convective inhibition in the modified sounding enabled storm-generated fluctuations to produce more numerical noise in the Dumas case compared to the Goshen case. Therefore, a larger domain $(200 \mathrm{~km} \times 200 \mathrm{~km})$ with lateral damping layers was ultimately employed to ensure the simulated storm was not unduly influenced by boundary reflections. Based on the observed wind profiles for this case, the upper bound for the lower-tropospheric wind modifications during BSS was chosen to be $4 \mathrm{~km}$ AGL.

\section{Goshen County supercell simulations}

The environment in southeast Wyoming on 5-6 June 2009 was characterized by a rather straight hodograph in the early afternoon, which gradually transitioned into a strongly curved hodograph in the early evening (Fig. 1). An upper-level ridge, initially situated over the central United States, propagated slowly eastward. Throughout the day, westerly flow aloft increased over the high plains, with large-scale lift provided by an upper-level vorticity maximum approaching the region. Slight backing and strengthening of the surface winds in response to surface cyclogenesis in central Wyoming contributed to additional hodograph curvature during the period of study, as well as upslope flow that aided in the initiation of convection (Markowski et al. (2012a), see their Fig. 1]; this is consistent with favorable environments for high plains severe thunderstorms reported in Doswell (1980). Convective cells were initiated north of Cheyenne, Wyoming, before 2000 UTC (Atkins et al. 2012, see their Fig. 1), and a well-documented tornadic supercell and EF2 tornado were well under way by 2200 UTC (e.g., Wakimoto et al. 2011; Atkins et al. 2012; Markowski et al. 2012a,b; Wakimoto et al. 2012; Wurman et al. 2012; Kosiba et al. 2013; Marquis et al. 2014). The supercell continued to move eastward into western Nebraska by 0000 UTC. The observed VORTEX2 soundings from 2155 to 0057 UTC showed increases in the $1-\mathrm{km}$ bulk wind difference, $0-1-\mathrm{km}$ SRH, and $0-3-\mathrm{km} \mathrm{SRH}$ of approximately $6 \mathrm{~m} \mathrm{~s}^{-1}, 200 \mathrm{~m}^{2} \mathrm{~s}^{-2}$, and $250 \mathrm{~m}^{2} \mathrm{~s}^{-2}$, respectively [Table 1 (top)].

\section{a. Simulated convection and vorticity evolution}

To evaluate the effects of the observed shear increase on a mature supercell, simulations using the observed soundings from VORTEX2 were performed, as described in section 2. The lower-shear Control simulation, using the 2155 UTC sounding, produces supercellular convection as was observed (Fig. 3), although because of the idealized modeling setup, comparisons to the observed supercell are necessarily superficial. Both the lower-shear Control storm and the higher-shear BSS storm maintain "classic" supercell structures through the first $2.5 \mathrm{~h}$ after BSS (Fig. 3). Eventually, the outflow from the Control storm undercuts the updraft, leading to the demise of the control supercell and a more disorganized multicellular structure (not shown). The BSS storm remains an isolated, intense supercell throughout the simulation. The hookecho, weak-echo regions, and rightward propagation, all common radar features associated with supercells, were more pronounced in the highershear BSS supercell.

In the higher-shear storm, the vertical vorticity throughout the lower-levels of the storm (i.e., in the lowest $2-4 \mathrm{~km}$ AGL) increases by approximately $50 \%$ after BSS (Fig. 4). This indicates an overall strengthening of the low-level mesocyclone, as would be expected from the enhanced environmental SRH. Both the lowershear Control and higher-shear BSS supercells consistently produce appreciable near-surface vertical vorticity (greater than $0.04 \mathrm{~s}^{-1}$ ). However, the higher-shear BSS supercell produces a near-surface vortex that strengthens to almost $0.2 \mathrm{~s}^{-1}$ soon after the completion of the BSS process, which is twice as strong as any produced by the Control storm. These results are particularly interesting because, as mentioned earlier, the factors that control the amplification of surface vorticity are still unclear. The low-level wind profile is the only element that differs between the two model runs. Even though these simulations are not tornado resolving, it appears that simply elongating the hodograph in the low levels dramatically alters the development of strong surface vorticity.

\section{b. Updraft intensification}

The higher-shear BSS storm has a stronger maximum updraft throughout the lower and middle troposphere, by roughly $10 \mathrm{~m} \mathrm{~s}^{-1}$ (Fig. 5). The core of the updraft (vertical velocities greater than $20 \mathrm{~m} \mathrm{~s}^{-1}$ ) descends to lower altitudes (Fig. 5), and also acquires a greater areal extent over time (Fig. 6). These changes imply that both the footprint and magnitude of the low-level vertical accelerations have been enhanced by the increase in shear. Since environmental buoyancy was unchanged during BSS, it seems most likely that these updraft differences are either due to the direct dynamical effects of enhanced shear, or due to emerging secondary effects associated with differences in the simulated cold pools. The preponderance of evidence suggests that the updraft enhancement was not caused by enhanced cold pool lifting. In our simulations, the averaged potential temperature perturbation of the outflow was actually slightly smaller in the higher-shear BSS run (Fig. 7), with 
Goshen Lower Shear $t=5400 s$

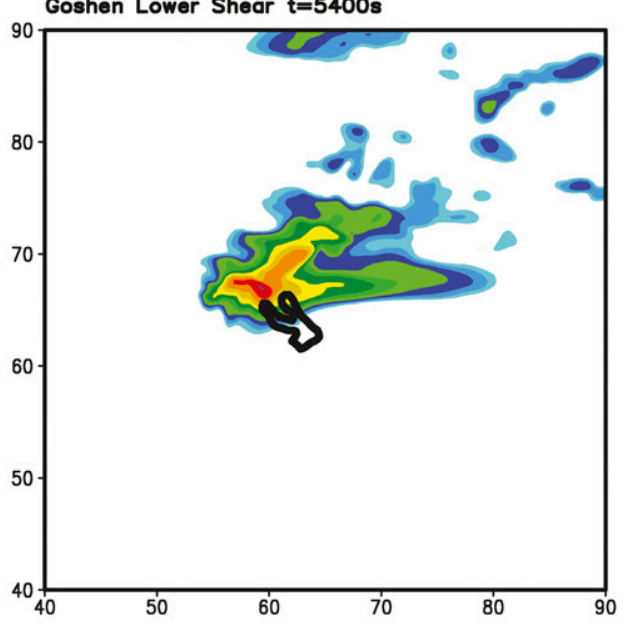

Goshen Lower Shear $t=9000 s$
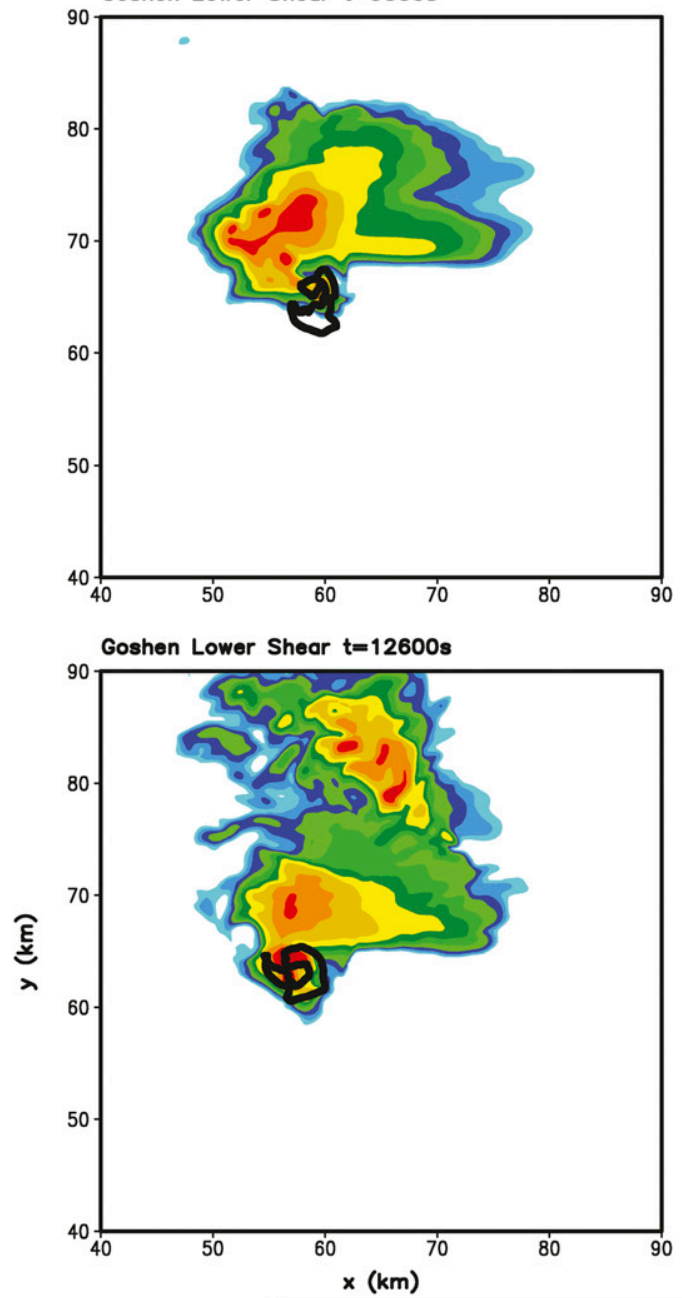

Goshen Higher Shear $t=5400 s$ (Start of BSS)

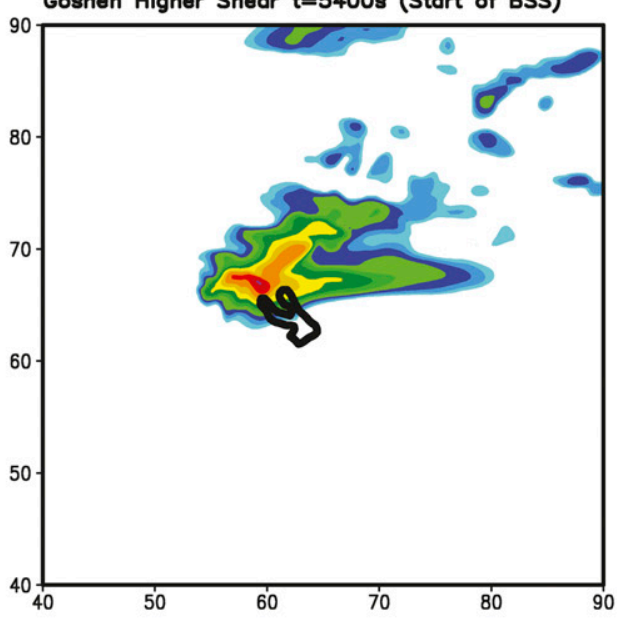

Goshen Higher Shear $t=9000$ s (End of BSS)

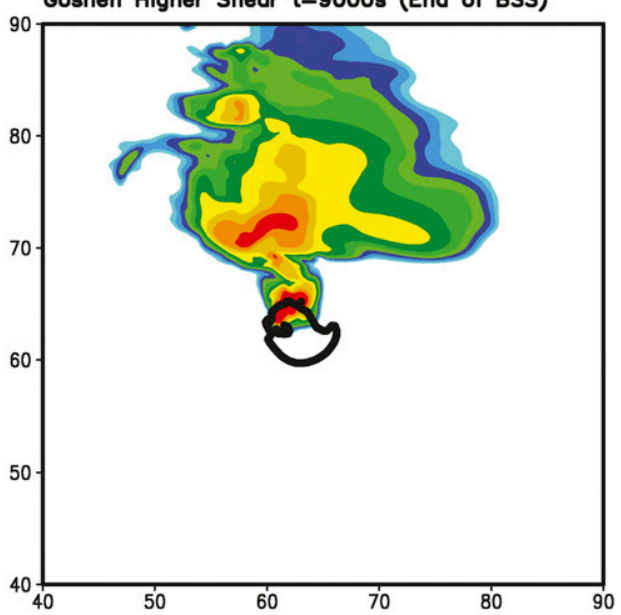

Goshen Higher Shear $t=12600 \mathrm{~s}$ (End of BSS $+1 \mathrm{hr}$ )

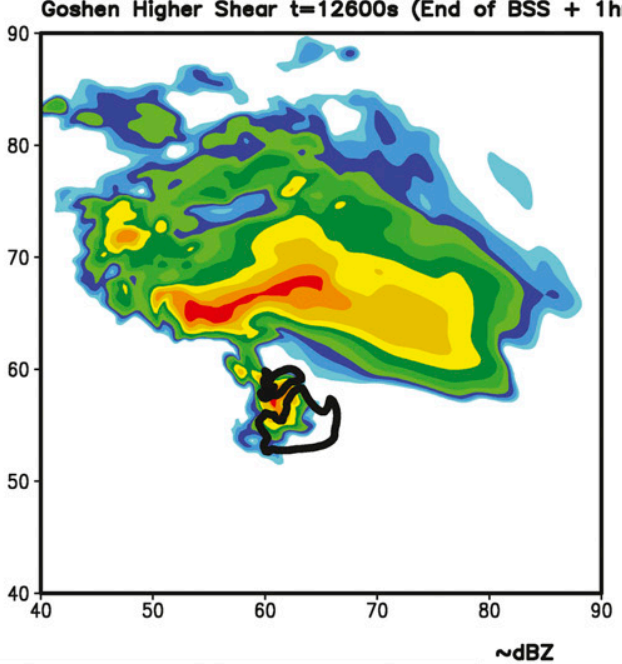

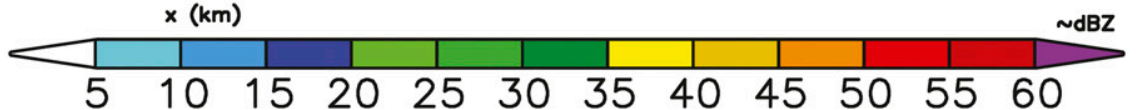

FIG. 3. Model-simulated reflectivity at $500 \mathrm{~m}$ for (left) the lower-shear Goshen Control simulation and (right) the higher-shear Goshen BSS simulation at the start of BSS, the end of BSS, and $1 \mathrm{~h}$ after BSS. The $500 \mathrm{~m}^{2} \mathrm{~s}^{-2} 1-6-\mathrm{km}^{2}$ updraft helicity [defined by Kain et al. (2008)] contour is shown in black. The wind profile in the BSS simulation evolved from the Control, to the BSS1, and finally to the BSS2 soundings. 

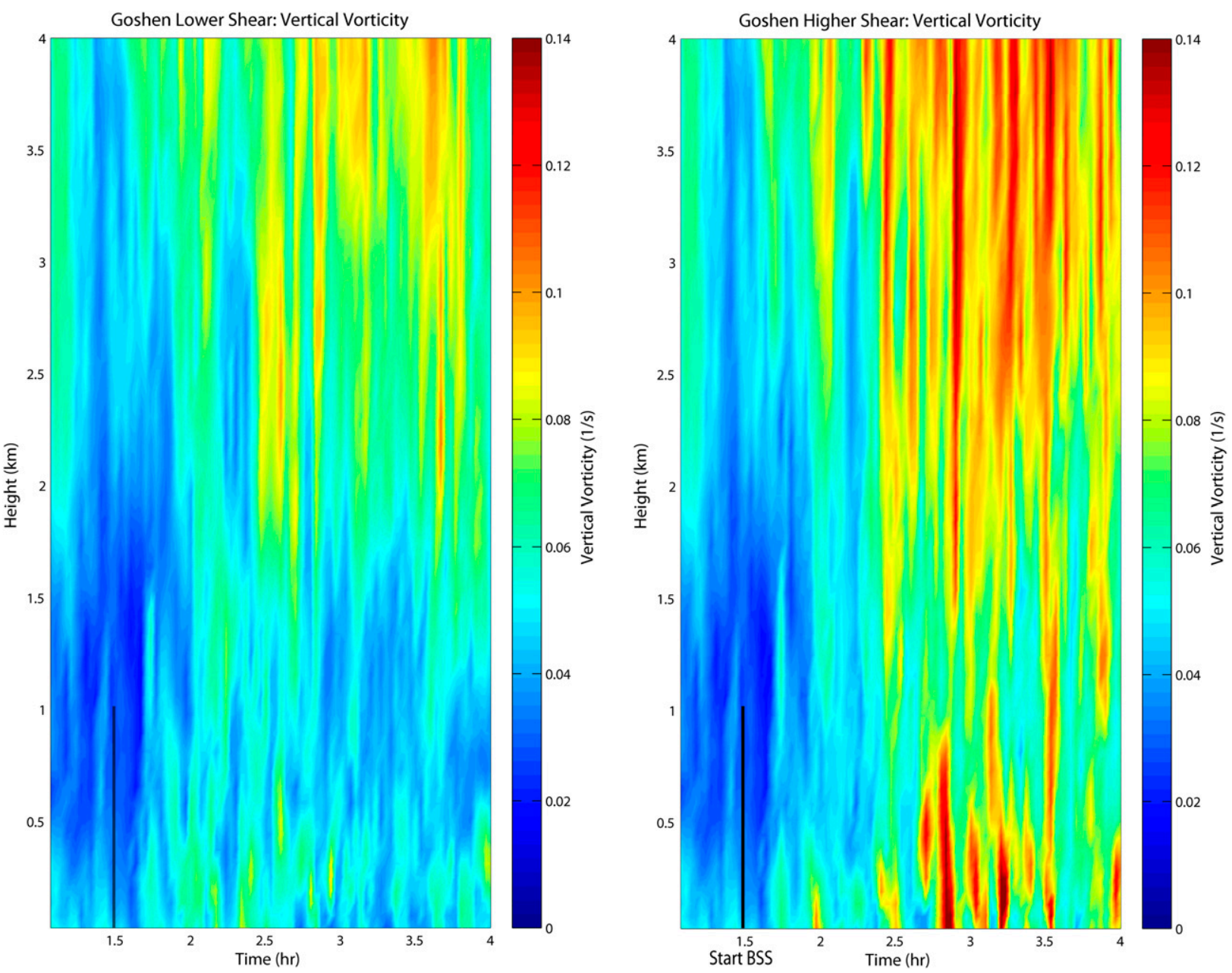

FIG. 4. Time-height plot of the maximum vertical vorticity $\left(\mathrm{s}^{-1}\right)$ in a $50 \mathrm{~km} \times 50 \mathrm{~km}$ box following the supercell's mesocyclone for both (left) the lower-shear Goshen Control simulation and (right) the higher-shear Goshen BSS simulation. The BSS process for the highershear simulation begins at $1.5 \mathrm{~h}$, indicated by the dark black line. Although no changes to the wind profile were made in the lower-shear simulation, a faded black line was placed at $1.5 \mathrm{~h}$ for reference.

negligible differences in the immediate vicinity of the near-surface vorticity maxima (gray "blob" in Fig. 7).

Because the cold pool hypothesis was not explanatory, we next assessed the lifting of air due to the dynamic component of the vertical pressure perturbation gradient acceleration (VPPGA). Changes in the dynamic VPPGA were calculated for both the 0-1- and 0-3-km layers in a $50 \mathrm{~km} \times 50 \mathrm{~km}$ box following the supercell's mesocyclone. To support our dynamical analysis, we calculate the components of the perturbation pressure $p^{\prime}$ for each model output time. The total $p^{\prime}$ field is known from the original model output. Following Wilhelmson and Ogura (1972) and Rotunno and Klemp (1982), the buoyant and dynamic components of the pressure perturbation $\left(p_{B}^{\prime}+p_{D}^{\prime}=p^{\prime}\right)$ can be diagnosed using

$$
\nabla^{2} p_{B}^{\prime}=\frac{\partial}{\partial z}\left(\rho_{o} B\right)
$$

and

$$
\begin{aligned}
\nabla^{2} p_{D}^{\prime}= & -\rho_{o}\left[\left(\frac{\partial u}{\partial x}\right)^{2}+\left(\frac{\partial v}{\partial y}\right)^{2}+\left(\frac{\partial w}{\partial z}\right)^{2}\right] \\
& -w^{2} \frac{\partial^{2}}{\partial z^{2}}\left(\ln \rho_{o}\right) \\
& -2 \rho_{o}\left(\frac{\partial v}{\partial x} \frac{\partial u}{\partial y}+\frac{\partial u}{\partial z} \frac{\partial w}{\partial x}+\frac{\partial v}{\partial z} \frac{\partial w}{\partial y}\right) .
\end{aligned}
$$

By separating the velocities into mean and perturbation parts, one can further isolate the linear component of (2), which is

$$
\nabla^{2} p_{\mathrm{DL}}^{\prime}=-2 \rho_{o}\left(\frac{\partial u_{o}}{\partial z} \frac{\partial w}{\partial x}+\frac{\partial v_{o}}{\partial z} \frac{\partial w}{\partial y}\right)
$$

The interpretation of these components can be simplified by employing the formulation described by 

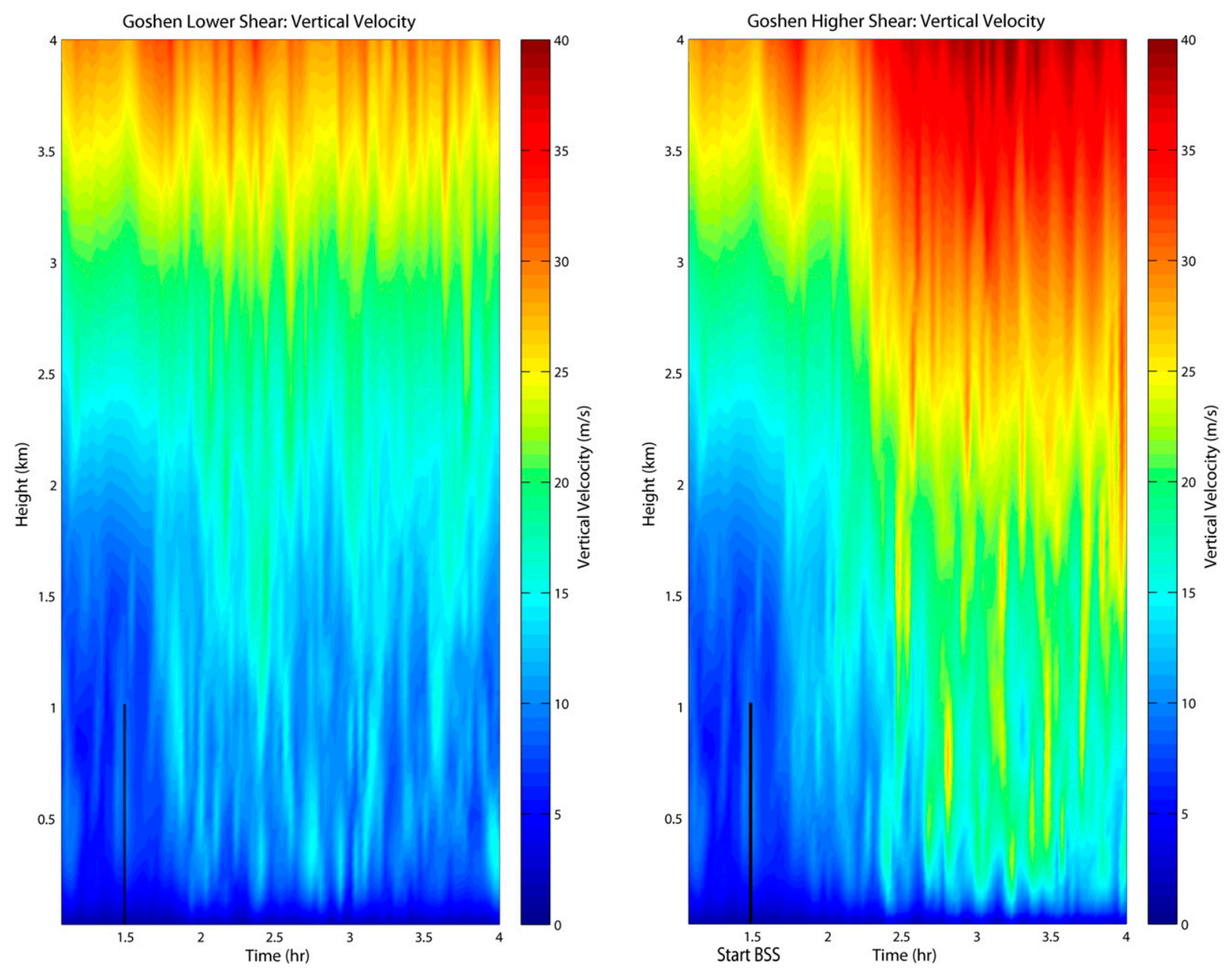

FIG. 5. As in Fig. 4, but for the maximum vertical velocity $\left(\mathrm{m} \mathrm{s}^{-1}\right)$.

Markowski and Richardson (2010, p. 30), where for well-behaved, incompressible, storm-scale flows, $p^{\prime}$ is approximately given by

$$
p^{\prime} \propto \underbrace{e_{i j}^{\prime 2}-\frac{1}{2}\left|\boldsymbol{\omega}^{\prime}\right|^{2}}_{\text {nonlinear dynamic }}+\underbrace{2 \overline{\mathbf{S}} \cdot \nabla_{h} w^{\prime}}_{\text {linear dynamic }}-\underbrace{\frac{\partial B}{\partial z}}_{\text {buoyant }} .
$$

The first two terms on the right-hand side (rhs) are the nonlinear dynamic pressure perturbation terms, including the deformation of the perturbation wind (deformation tensor: $e_{i j}$ ) and the total vorticity of the perturbation wind $(\boldsymbol{\omega})$. Deformation and/or divergence (commonly referred to as "splat") are associated with positive pressure perturbations. Rotation (commonly referred to as "spin") is associated with low pressure. The third term on the rhs is the linear dynamic pressure perturbation term, and is associated with an updraft $(w)$ in environmental wind shear (S). Low (high) pressure is found downshear (upshear) of an existing updraft. The final term on the rhs is the buoyancy $(B)$ pressure perturbation term.

For our calculations, we solve (1)-(3) iteratively in the interior of the domain using the following boundary conditions: $p_{B}^{\prime}$ [buoyant pressure perturbation; (1)] on all boundaries satisfies the hydrostatic equation, and $p_{D}^{\prime}$ [total dynamic pressure perturbation; (2)] on all boundaries satisfies $p_{D}^{\prime}=p^{\prime}-p_{B}^{\prime}$. Once we have solved for $p_{D}^{\prime}$, we then solve iteratively for $p_{\mathrm{DL}}^{\prime}$ [linear dynamic pressure perturbation; (3)] using the condition $p_{\mathrm{DL}}^{\prime}=$ 0 on the lateral boundaries, and then treat $p_{\mathrm{DNL}}^{\prime}$ (nonlinear pressure perturbation) as the residual: $p_{\mathrm{DNL}}^{\prime}=$ $p_{D}^{\prime}-p_{\mathrm{DL}}^{\prime}$. Once all pressure perturbation components are retrieved on all grid points, we then calculate the 

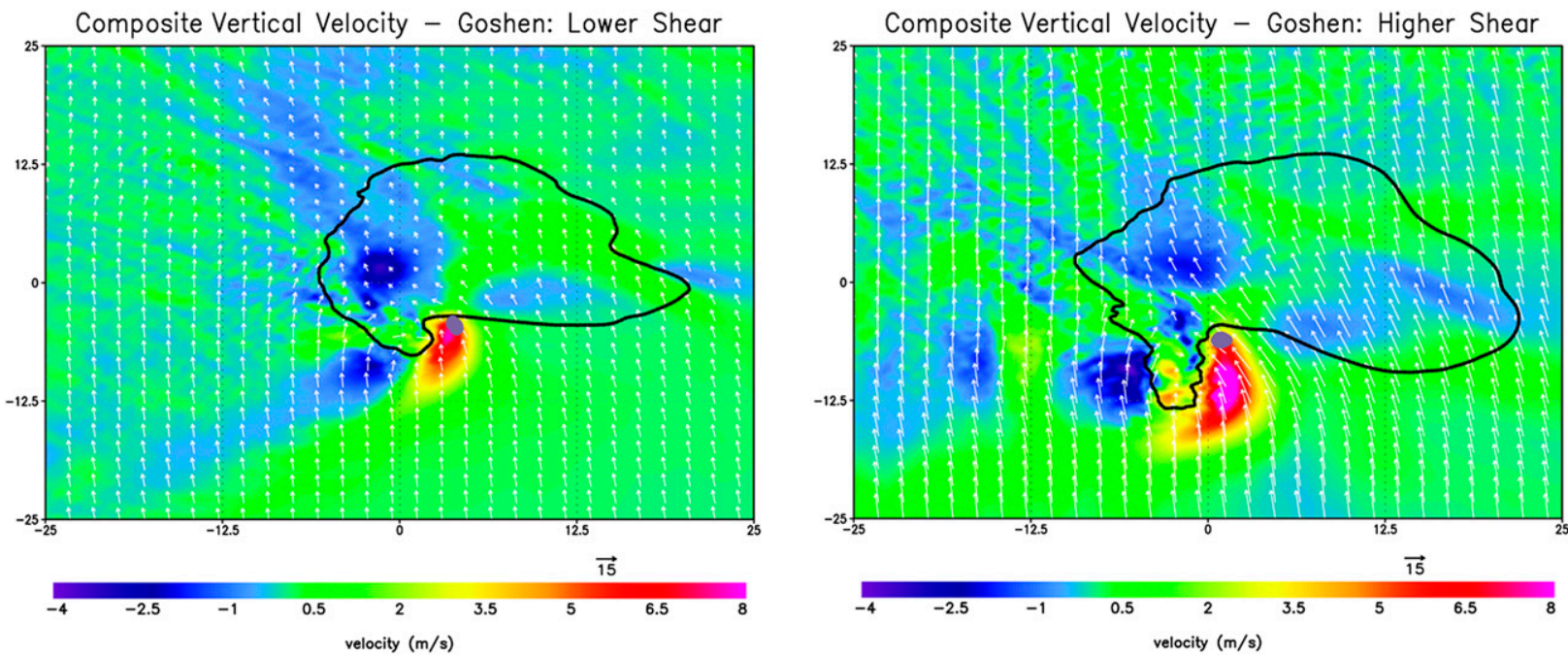

FIG. 6. Time composite plot showing the average spatial 1-km vertical velocity field $\left(\mathrm{m} \mathrm{s}^{-1}\right)$ for the hour following the end of the BSS process for (left) the lower-shear Goshen Control and (right) the higher-shear Goshen BSS simulation. The 20-dBZ composite reflectivity contour is shown in black. The average location of surface vertical vorticity maximum $\left(0.005 \mathrm{~s}^{-1}\right)$ is shown as a gray "blob." Composite, ground-relative $1-\mathrm{km}$ wind vectors are shown in white. Time composite plots were calculated by averaging values in a $50 \mathrm{~km} \times 50 \mathrm{~km}$ box following the supercell's mesocyclone [determined by the maximum 1-6-km updraft helicity; Kain et al. (2008)].

necessary vertical derivatives to produce the accelerations that we report. The VPPGA is simply $\left(-1 / \rho_{o}\right) \partial p^{\prime} / \partial z$, and the components of the VPPGA are the parts of the VPPGA associated with $p_{\mathrm{B}}^{\prime}, p_{D}^{\prime}, p_{\mathrm{DL}}^{\prime}$, and $p_{\mathrm{DNL}}^{\prime}$. Notably, the total buoyant acceleration includes both the buoyant VPPGA associated as well as the vertical acceleration due to buoyancy term itself. It has long been known that the dynamic accelerations can be quite large in supercells (e.g., Rotunno and Klemp 1982), occasionally even exceeding the upward accelerations due to buoyancy (e.g., McCaul and Weisman 1996).

In terms of both the box-maximum and box-averaged values, a much stronger dynamic VPPGA was observed at low levels in the higher-shear BSS storm compared to the lower-shear Control storm (Fig. 8). The maximum values in both the $0-1-$ and $0-3-\mathrm{km}$ layers increases by approximately $0.1 \mathrm{~m} \mathrm{~s}^{-2}$, and the areal extent of intense $0-1-\mathrm{km}$ VPPGA values also greatly increases as the storm encounters higher environmental low-level shear
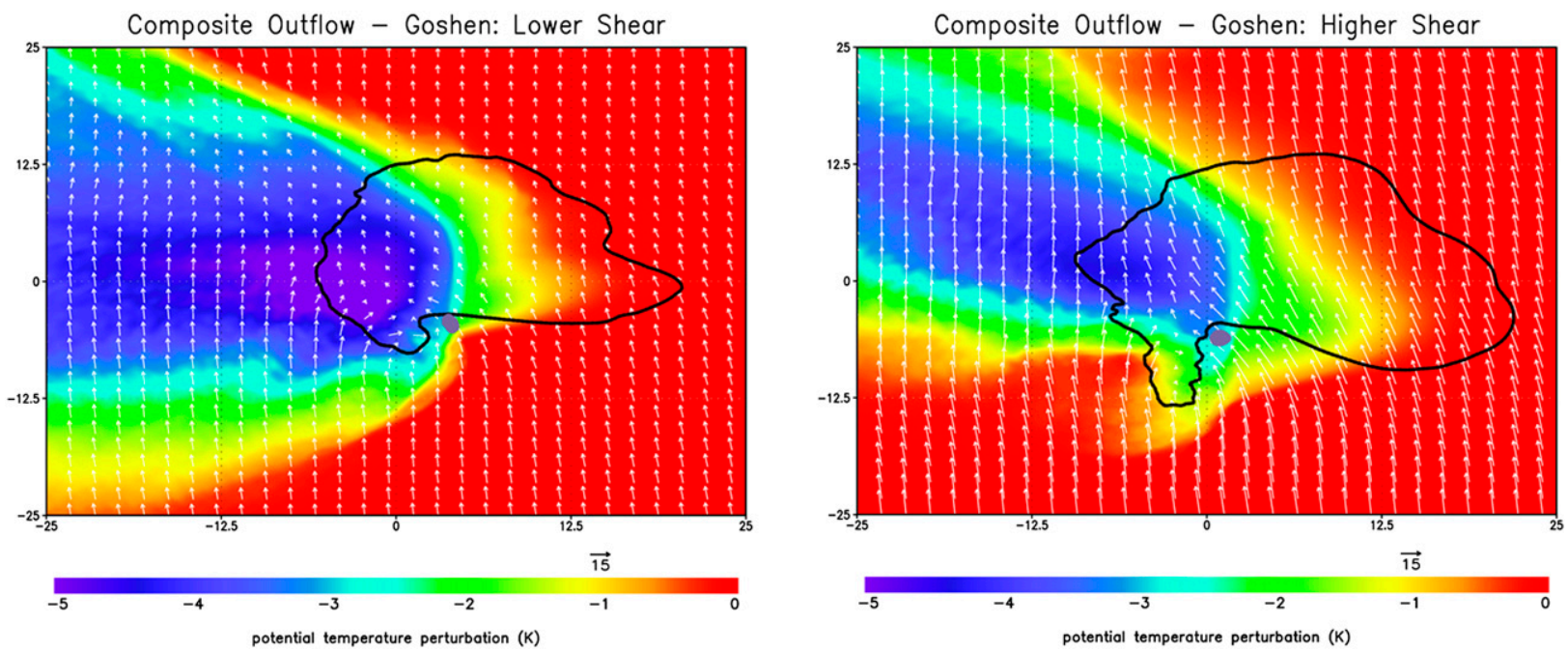

FIG. 7. As in Fig. 6, but for the surface potential temperature perturbation (K) 

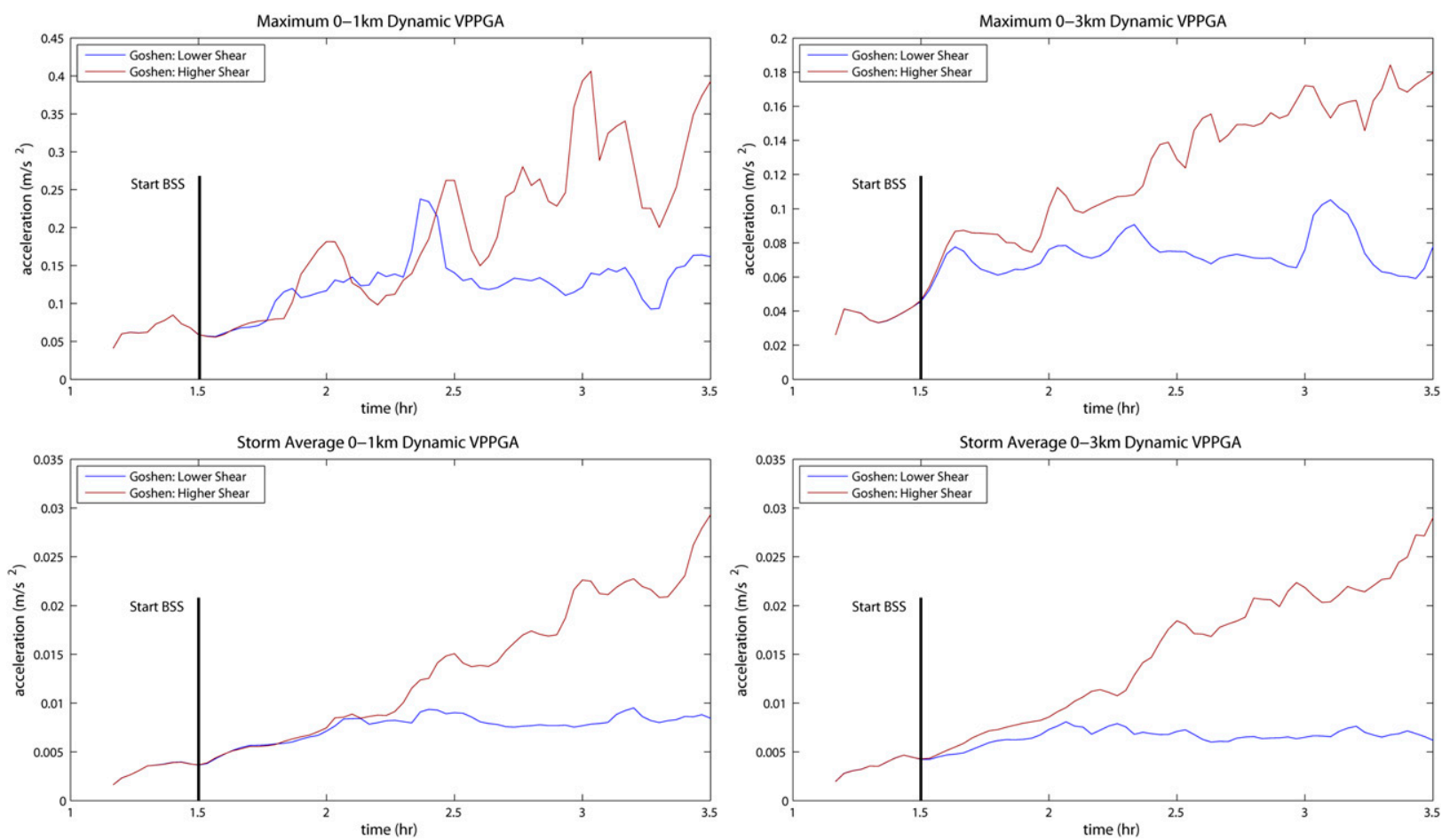

FIG. 8. Time series comparing the dynamic VPPGA $\left(\mathrm{m} \mathrm{s}^{-2}\right)$ for both the lower-shear Goshen Control (blue) and the higher-shear Goshen BSS (red) supercells. (top) The maximum acceleration value for both the 0-1- and 0-3-km layers in a $50 \mathrm{~km} \times 50 \mathrm{~km}$ box following the supercell's mesocyclone. (bottom) As in (top), but for the storm-averaged acceleration in the $50 \mathrm{~km} \times 50 \mathrm{~km}$ box. The BSS process for the higher-shear simulation begins at $1.5 \mathrm{~h}$, indicated by the dark black line.

(Fig. 9). The buoyant accelerations remained steady throughout the BSS process and values were generally 4 times lower than dynamic VPPGA (see Fig. S1 in the online supplemental material). Given that CAPE was unchanged across the experiments, it is probably not surprising that the changes to the buoyant accelerations were small, and generally dominated by the dynamical effects of shear.

What is the primary reason for the enhanced dynamic accelerations? The supercell mesocyclone's large vertical vorticity is associated with locally minimized pressure aloft via the second ("spin") term on the rhs of (4).
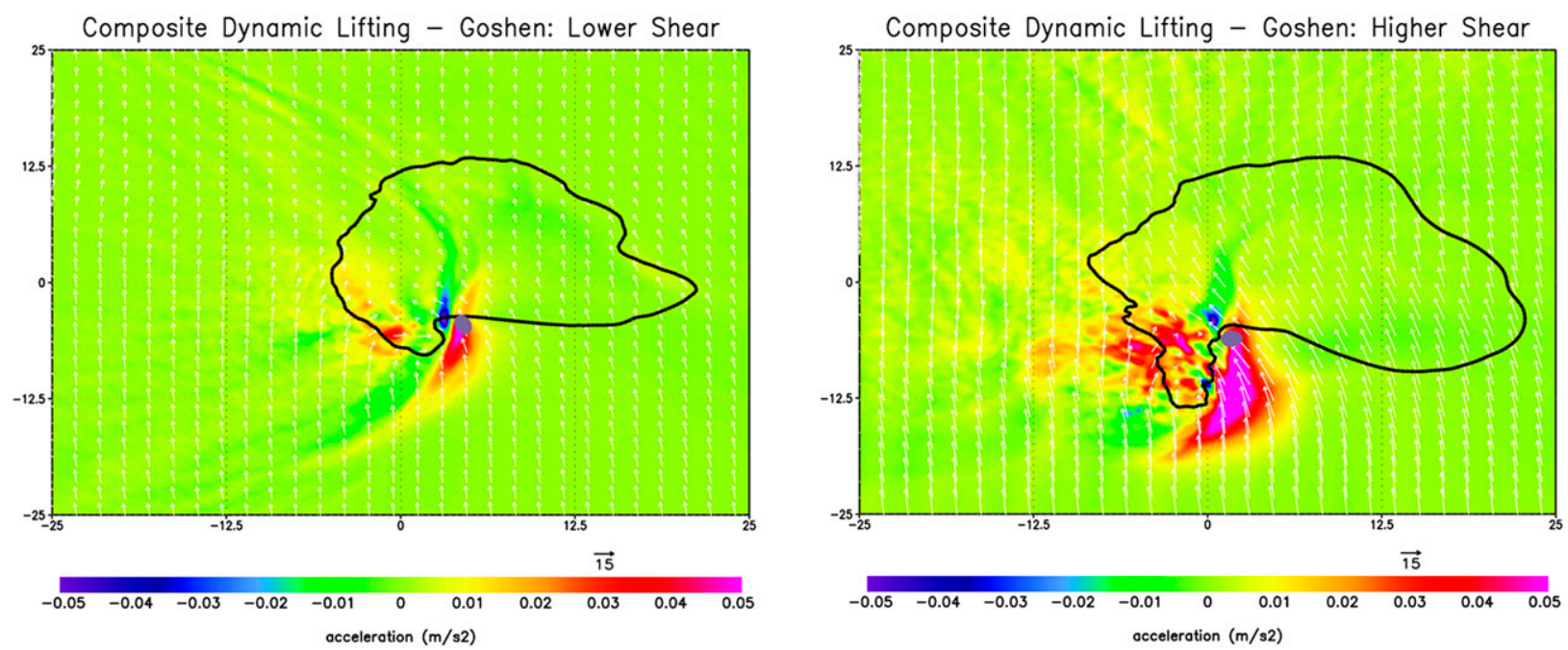

FIG. 9. As in Fig. 6, but for the 0-1-km dynamic VPPGA $\left(\mathrm{m} \mathrm{s}^{-2}\right)$. 


\section{Bulk Characteristics for Outflow Trajectories: Goshen}
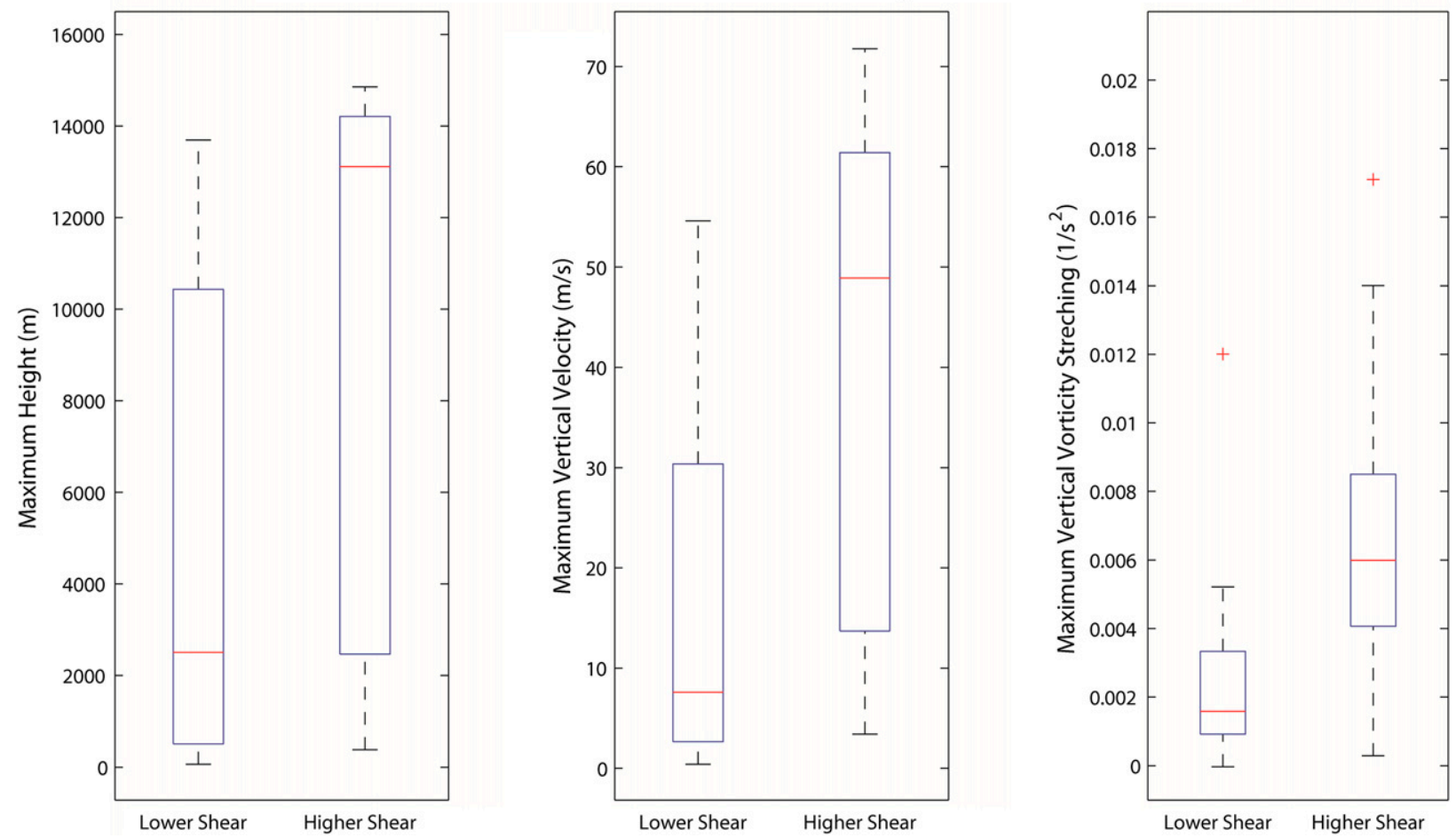

FIG. 10. Box plot showing (left) the maximum attained height $(\mathrm{m})$, (middle) vertical velocity $\left(\mathrm{m} \mathrm{s}^{-1}\right)$, and (right) maximum vertical velocity stretching $\left(\mathrm{s}^{-2}\right)$ for the outflow trajectories of both the lower-shear Goshen Control and higher-shear Goshen BSS supercell. All trajectories acquired at least $0.03 \mathrm{~s}^{-1}$ vertical vorticity and a $-2.5-\mathrm{K}$ potential temperature perturbation at the bottom model level. Trajectories were filtered to have the same initial buoyancy. The boxes represent the 25th and 75 th percentile, the whiskers denote approximately $\pm 2.7 \sigma$, and the red plus signs indicate any outliers.

One important effect of increased environmental vertical wind shear at low levels may be to establish the base of the low-level mesocyclone at lower altitudes. For comparable updraft structure, more horizontal vorticity yields faster tilting rates. This idea was presented by Markowski et al. (2012b), who analyzed observed mobile radar data from the same "Goshen County" VORTEX2 case (5 June 2009), and supported by idealized simulations of "pseudostorms" in Markowski and Richardson (2014). In the present study, this lowering of the base of the mesocyclone can be seen in both Figs. 4 and 5 . Increasing values of vertical vorticity aloft can then feedback on the upward dynamic VPPGA through enhanced perturbations associated with the spin term in (4). Calculations reveal that the intensification of the dynamic forcing is dominated by these nonlinear dynamic pressure perturbations (time-averaged $0-1-\mathrm{km}$ maximum: $0.26 \mathrm{~m} \mathrm{~s}^{-2}$ ), as opposed to the linear updraftin-shear effect (time-averaged $0-1-\mathrm{km}$ maximum: $0.05 \mathrm{~m} \mathrm{~s}^{-2}$ ). There is also a compounding effect of this nonlinear interaction. Since increased shear strengthens the updraft, this further enhances the tilting and stretching rates, which can generate even stronger vertical vorticity (and dynamic pressure minimization) at low levels. This is possibly why the storm-averaged dynamic VPPGA in Fig. 8 shows an increasing trend with time.

\section{c. Trajectory analysis}

The final step in the tornadogenesis process involves converging and stretching surface vertical vorticity into a tornadic circulation. Although the present simulations are not tornado resolving, we still wished to evaluate the ingestion of vorticity-rich outflow air by the supercell's overlying updraft. Several million parcels were released in the cold pool of both the lower- and higher-shear Goshen simulations $15 \mathrm{~min}$ after the BSS process was completed. Forward trajectories were integrated natively on the model time step (2s) in CM1, with output saved every $10 \mathrm{~s}$ for a period of $30 \mathrm{~min}$. To focus on outflow parcels with appreciable vertical vorticity, we analyzed the subset of the trajectories that had a temperature deficit of at least $-2.5 \mathrm{~K}$ and vertical vorticity of at least $0.03 \mathrm{~s}^{-1}$ at the bottom model level. Despite similar initial buoyancy, approximately $40 \%$ more outflow parcels acquired appreciable near-surface vorticity 


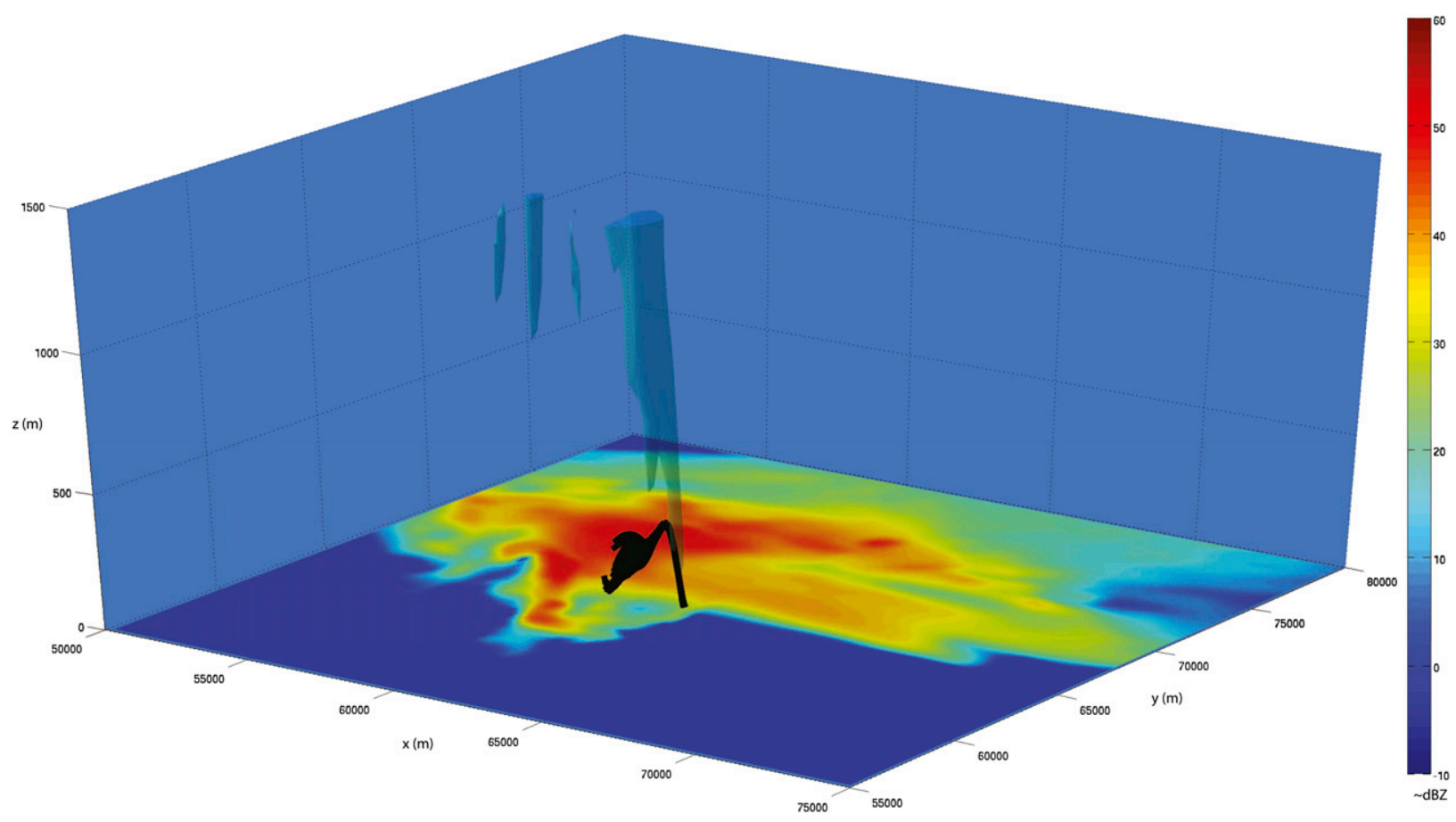

FIG. 11. Three-dimensional view of the low-level updraft, near-surface reflectivity field, and the 20 near-surface vortex trajectories (black) that acquired the most surface vorticity at the bottom model level in the lower-shear Goshen Control supercell. Shaded on the surface is the model-simulated base reflectivity $(\sim \mathrm{dBZ})$. Updraft isosurfaces of 9 and $18 \mathrm{~m} \mathrm{~s}^{-1}$ are shown light green and light red, respectively (if present).

in the higher-shear BSS supercell (1004 vs 619). Comparing outflow parcels with identical initial buoyancy shows that outflow parcels in the higher-shear BSS supercell generally rose to much greater heights and experienced greater vertical velocities and stretching of vorticity than their counterparts in the lower-shear Control supercell (Fig. 10).

An additional set of trajectories was launched when both simulations were producing a near-surface vortex $(t \approx 2.8 \mathrm{~h})$. To determine the fate of the parcels that entered the developing vortex, these parcels were densely launched in $3 \mathrm{~km} \times 3 \mathrm{~km} \times 1 \mathrm{~km}$ box surrounding the vortex and integrated forward until the vortex dissipated. Trajectories were filtered based on which vortex parcels acquired the most vertical vorticity at the bottom model level. Shown in Figs. 11 and 12 are the 20 vortex parcels that acquired the most vertical vorticity at the bottom model level in each simulation. The vortex trajectories that acquired large near-surface vorticity often stagnated in the lower-shear Control supercell and were advected toward the rear of the storm (Fig. 11), instead of rising vertically and participating in the overlying updraft, as in the higher-shear BSS supercell (Fig. 12). The vortex trajectories in the lower-shear Control supercell are indicative of a decoupling of surface cyclone from the parent updraft and mesocyclone, and thus a cessation or disruption of the vortex stretching (Trapp 1999). Similar trajectory behavior has been observed in nontornadic supercells (Markowski et al. 2011, see their Fig. 7).

Bulk statistics for the vortex trajectories shed light on why the near-surface vorticity in the higher-shear BSS simulation strengthened to almost double that of the lower-shear Control simulation (e.g., Fig. 4). Despite similar outflow temperatures (within $1 \mathrm{~K}$ ), parcels in the higher-shear BSS storm rose faster and higher, increasing the stretching of vorticity (Fig. 13). Even though the trajectories in the higher-shear supercell were roughly $1 \mathrm{~K}$ warmer, the differences in mean buoyant accelerations experienced by the parcels were small; however, the mean dynamic acceleration was almost 3 times as high in the higher-shear BSS storm (Fig. 13). Thus, it is likely that the near-surface vortex in the higher-shear BSS supercell strengthened considerably more than the lower-shear Control supercell because of the enhanced vertical stretching from the stronger dynamic VPPGA at low levels (as was described in Figs. 8 and 9).

It is worth briefly mentioning the volatility of nearsurface vorticity development in these simulations. The vortices that develop are small in scale $(<1 \mathrm{~km})$ and rarely last more than a few minutes. Additionally, 


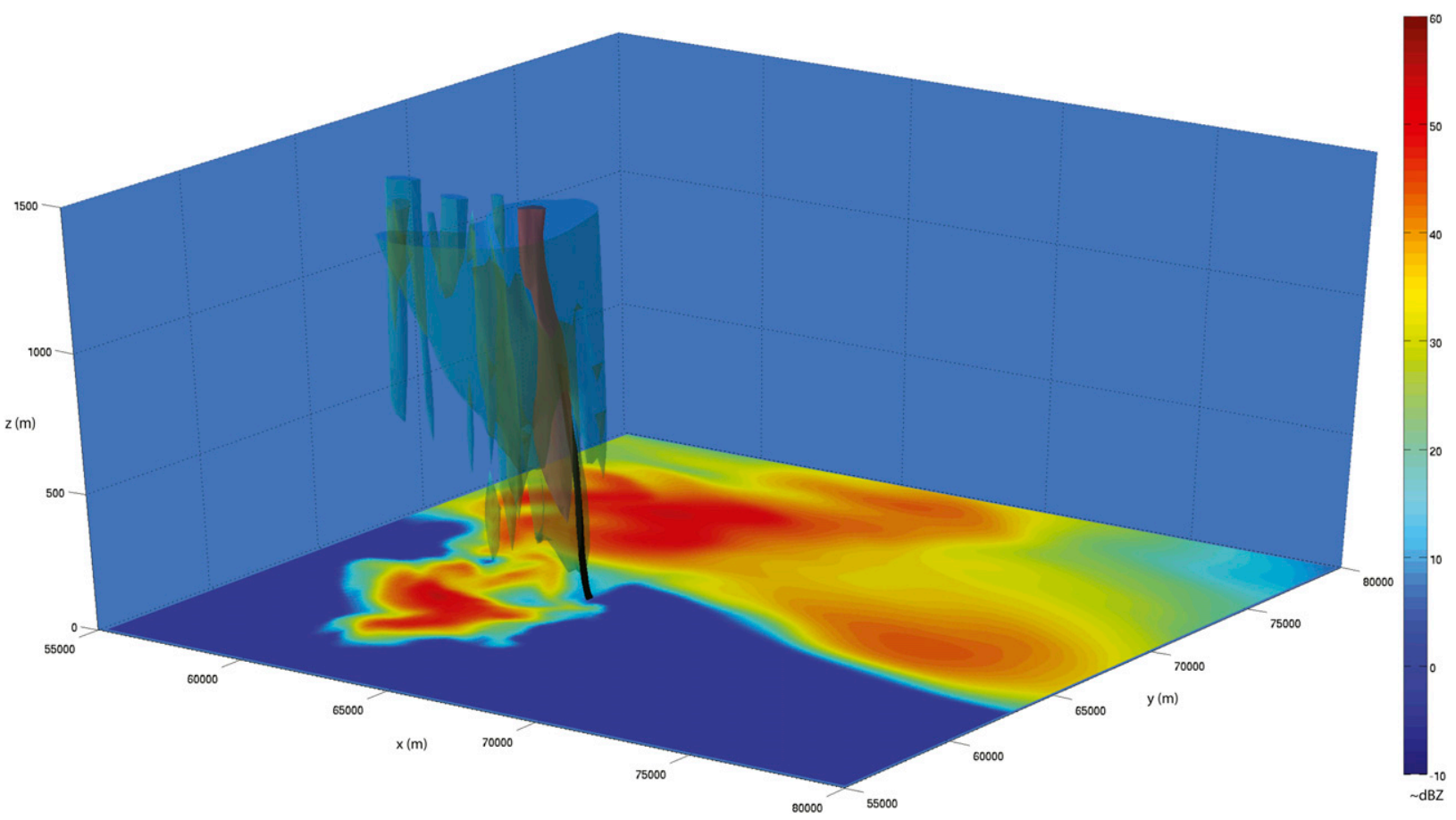

FIG. 12. As in Fig. 11, but displayed is the higher-shear Goshen BSS supercell.

seemingly minor changes in the initial conditions and model setup can cause deviations in the evolution of near-surface vorticity due to the highly nonlinear nature of vortex development. This volatility could be due to numerical procedures and settings, including the nontornado resolving $250-\mathrm{m}$ grid spacing and the lack of surface drag. Since the most intense surface vortices in this study only span a few grid points, they are heavily diffused. Even interpolating the horizontal velocity components to the scalar grid points reduces the magnitude of the maximum near-surface vorticity by a factor of 2. Additionally, the exclusion of surface drag prevents near-surface vortices from fully contracting into a tornado [i.e., including drag would disrupt cyclostrophic balance and induce strong radial inflow, as reviewed by Davies-Jones (2015)]. Because of these limitations, we do not want to attach excessive importance to the details of individual vortices; it is the substantial bulk differences and trends in the low-level vorticity and updraft fields that we view as most informative. The dramatically stronger dynamic lifting of vorticity-rich outflow air in the higher-shear BSS simulation would generally appear to be a much more favorable situation for realizing tornadogenesis compared to the lower-shear control simulation.

\section{d. Thermodynamic and stabilization experiments}

To isolate the role of the low-level wind profile in the preceding simulations, no thermodynamic changes were implemented during the BSS process. However, in nature the evolution of the wind field during the EET is intrinsically linked to changes in the temperature profile. Another higher-shear BSS simulation was performed that reflected both the thermodynamic and kinematic transition observed in the VORTEX2 soundings, although the thermodynamic changes were rather small (see Figs. S2 and S3 in the online supplemental material). Even under the observed stabilization, the supercell's mesocyclone responded very similarly by intensifying, lowering in altitude, and expanding spatially due to increases in the nonlinear component of the dynamic VPPGA (see Fig. S4 in the online supplemental material).

Since the observed stability changes were quite small, a supplementary suite of simulations was performed using the artificial stabilization technique described by French and Parker (2010). Starting at the initial time of BSS $(t=1.5 \mathrm{~h})$, for both the lower-shear Control and higher-shear BSS supercells, isothermal layers were progressively created by cooling the surface temperature by 2,4 , and $8 \mathrm{~K}$ at a rate of $3 \mathrm{~K} \mathrm{~h}^{-1}$. The aim was to understand how much stabilization a supercell might endure while remaining surface based. This is similar to the question addressed by Nowotarski et al. (2011), except in this case, we assess the impacts of stabilization upon an already-mature supercell.

In both the lower- and higher-shear cases, the simulations maintained the visual appearance of supercellular 

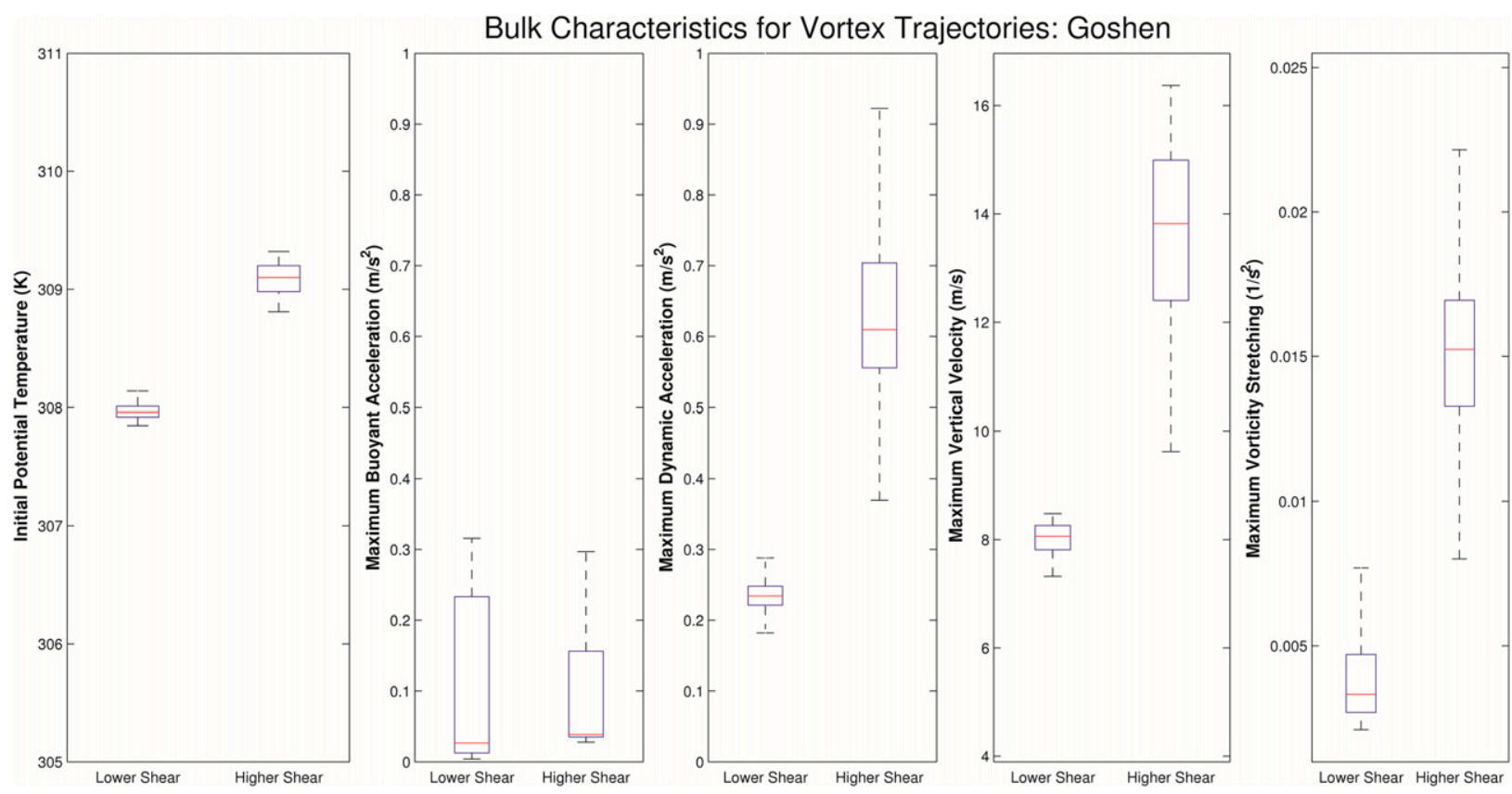

FIG. 13. Box plot showing the initial potential temperature (K), maximum buoyant acceleration $\left(\mathrm{m} \mathrm{s}^{-2}\right)$, maximum dynamic acceleration $\left(\mathrm{m} \mathrm{s}^{-2}\right)$, maximum vertical velocity $\left(\mathrm{m} \mathrm{s}^{-1}\right)$, and maximum stretching of vertical vorticity $\left(\mathrm{s}^{-2}\right)$ for the 20 near-surface vortex trajectories that acquired the most surface vorticity at the bottom model level in the lower-shear Goshen Control and higher-shear Goshen BSS supercell (as displayed in Figs. 11-12). The boxes represent the 25th and 75 th percentile, the whiskers denote approximately $\pm 2.7 \sigma$, and the red plus signs indicate any outliers.

features, including a hook-echo, weak-echo regions, and rightward propagation, regardless of the strength of the statically stable layer (not shown). The mesocyclone strength was reduced at low levels in the lower-shear Control supercell, but not until a significant amount of cooling had taken place (4K; see Fig. S5 in the online supplemental material). For the higher-shear BSS supercell, the positive influence of the increasing low-level shear on the mesocyclone was able to offset the negative influence of decreased surface buoyancy, with values of vertical velocity in excess of $20 \mathrm{~m} \mathrm{~s}^{-1}$ still regularly occurring below $2 \mathrm{~km}$ AGL (see Fig. S6 in the online supplemental material). The most noticeable difference between the simulations is in the development of nearsurface rotation. In the lower-shear Control supercell, the generation of appreciable near-surface vorticity ceases after cooling greater than $2 \mathrm{~K}$ (see Fig. S5 in the online supplemental material), whereas in the higher-shear BSS simulation near-surface values approaching $0.1 \mathrm{~s}^{-1}$ continue to occur even in the most strongly stabilized case (see Fig. S6 in the online supplemental materials). At most distances from conventional radars, the base scan is too high to detect rotation (or lack thereof) within the nocturnal boundary layer. This represents a considerable operational challenge during the EET, when low-level updraft and near-ground rotation may actually intensify as increases in shear predominate over low-level stabilization.

\section{Dumas supercell simulations}

The most robust results of the Goshen simulations are that observed increases in low-level shear lead to a more coherent supercell reflectivity structure, the enhancement of the low-level mesocyclone, and the subsequent increase in low-level dynamic lifting. Given our current understanding of tornadogenesis, these would all seem to favor the development of intense near-surface vortices, even though the development of near-surface vorticity in this model setup is somewhat volatile. To assess whether the Goshen results are representative, we next compare it (more briefly) to simulations of the 18-19 May 2010 Dumas tornadic supercell.

The evolution of the wind profile in the Texas Panhandle on 18-19 May 2010 was similar to the Goshen case. The hodograph during the late afternoon was predominately straight above $300 \mathrm{~m}$, and over time this evolved into a highly curved hodograph with a distinct increase in speed and veering of the winds below $2-3 \mathrm{~km}$ AGL during the early evening hours (Fig. 2). On 18 May a negatively tilted, upper-level trough was slowly approaching the central United States. During the afternoon, the mid-to-upper-level flow increased over the Texas and Oklahoma Panhandle region during the afternoon's peak heating, with mixed-layer CAPE values exceeding $2000 \mathrm{~J} \mathrm{~kg}^{-1}$. The VORTEX2 armada targeted 
Dumas Lower Shear $\mathrm{t}=5400 \mathrm{~s}$

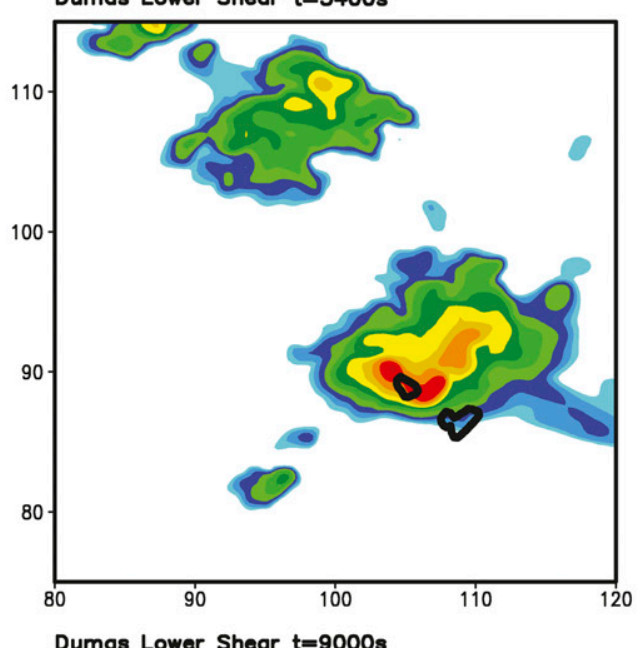

Dumas Lower Shear $t=9000 s$

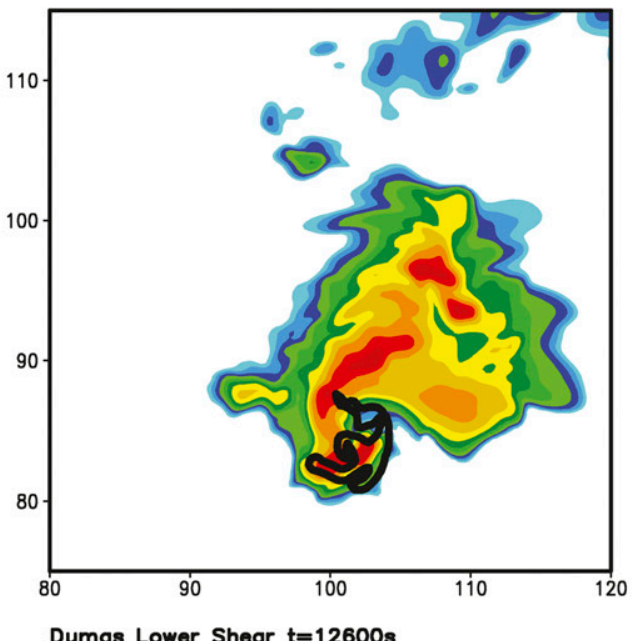

Dumas Lower Shear $t=12600$

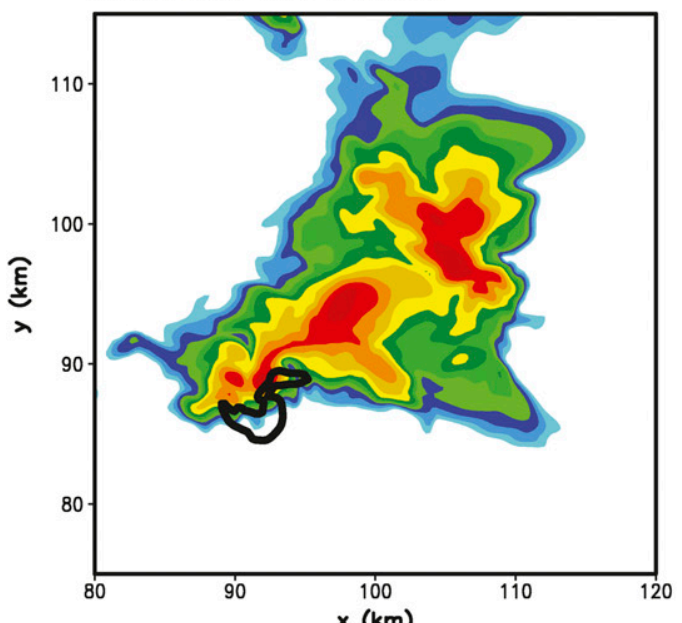

Dumas Higher Shear $t=5400 s$ (Start of BSS)
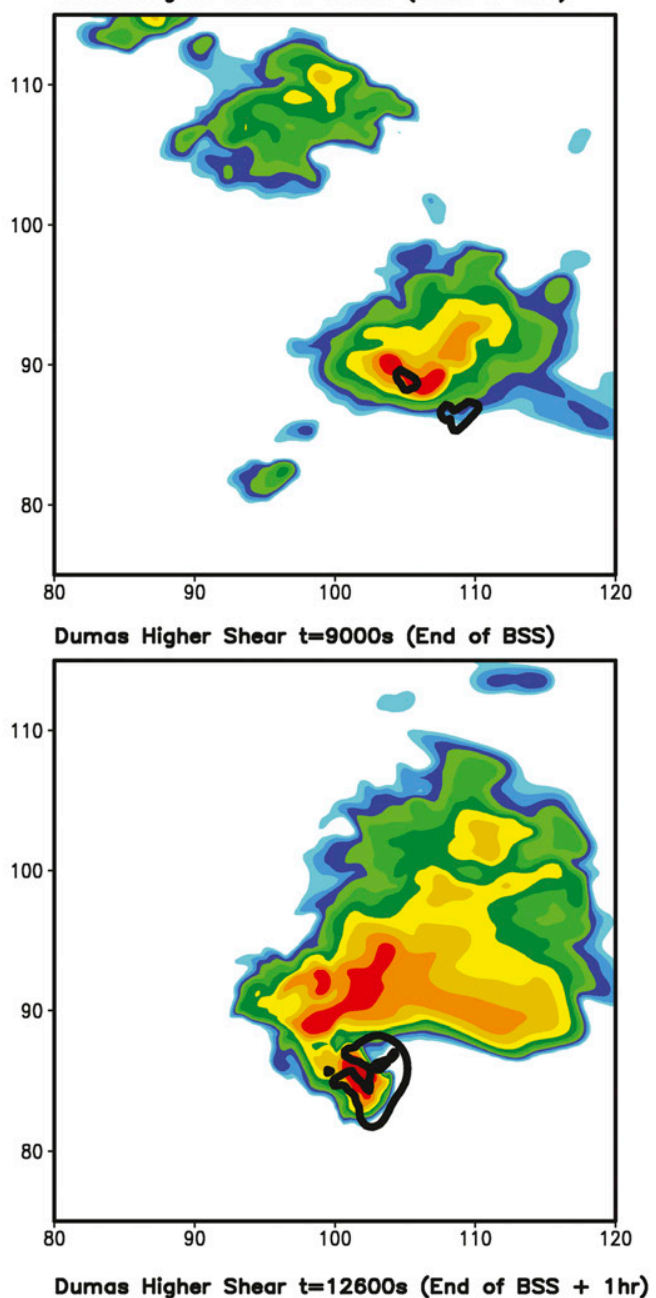

Dumas Higher Shear $t=12600$ s (End of BSS $+1 \mathrm{hr}$ )

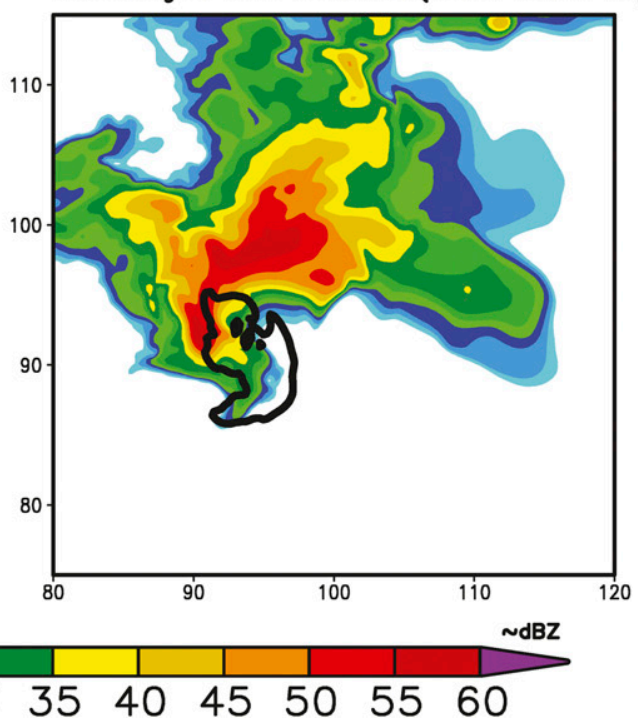

FIG. 14. As in Fig. 3, but for (left) the lower-shear Dumas Control simulation and (right) the higher-shear Dumas BSS simulation. 

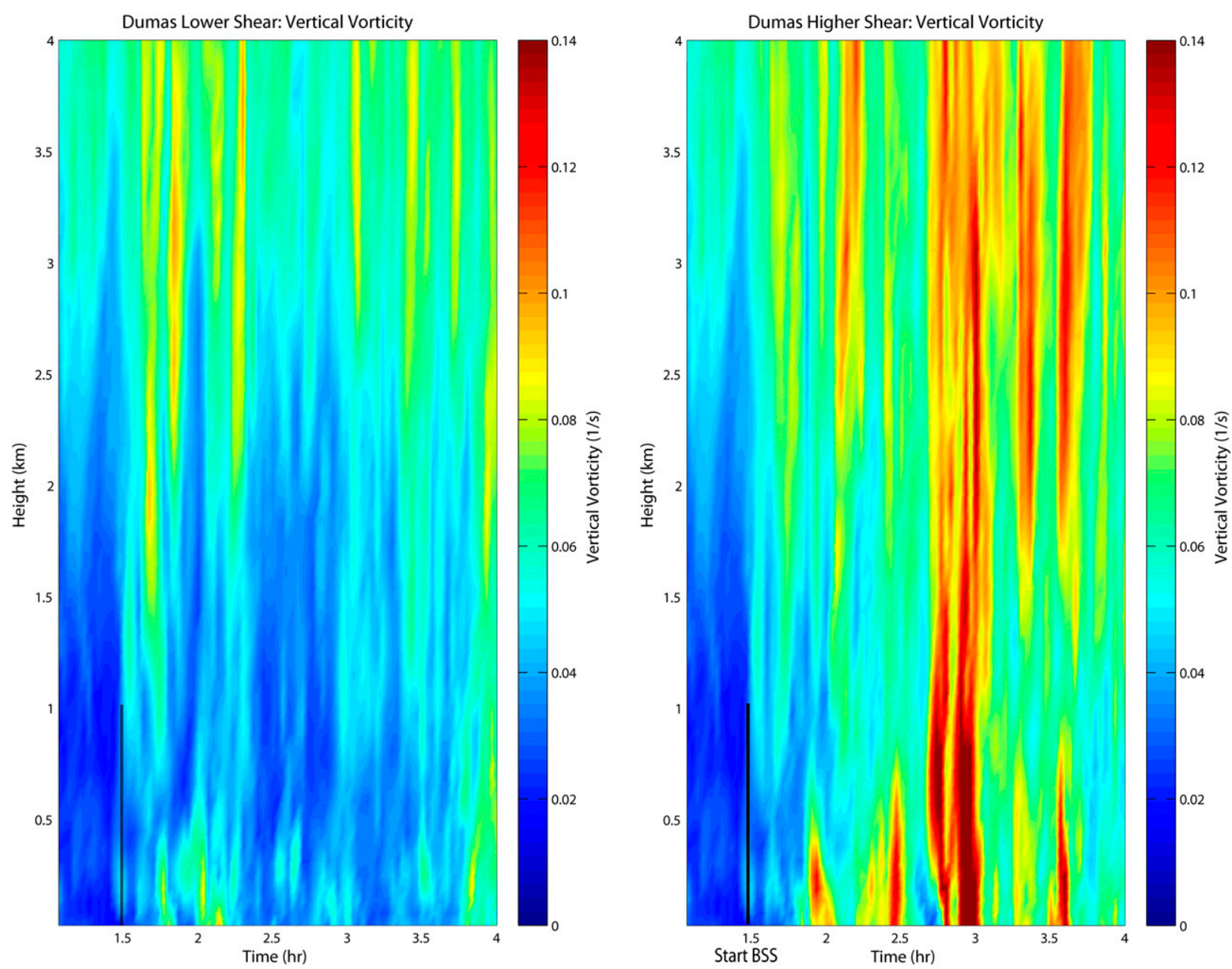

FIG. 15. As in Fig. 4, but for (left) the lower-shear Dumas Control simulation and (right) the higher-shear Dumas BSS simulation.

a developing supercell by 2200 UTC and continued sampling it as it moved east-northeastward across the Texas Panhandle. The supercell only managed to produce weak tornadoes from 2230 to 2330 UTC despite the visual presence of a strong low-level mesocyclone (Skinner et al. 2014; see their Figs. 4-5). As the supercell moved eastward, an EF2 tornado was documented at 0044 UTC (the same time as the first higher-shear BSS sounding was observed; Fig. 2). The observed VORTEX2 soundings from 2257 to 0130 UTC showed increases in the $1-\mathrm{km}$ bulk wind difference, $0-1-\mathrm{km}$ SRH, and $0-3-\mathrm{km}$ SRH of approximately $6 \mathrm{~m} \mathrm{~s}^{-1}, 200 \mathrm{~m}^{2} \mathrm{~s}^{-2}$, and $350 \mathrm{~m}^{2} \mathrm{~s}^{-2}$, respectively [Table 1 (middle)]. This case, therefore, complements the Goshen supercell study, having impressive low-level shear increases within an environment with slightly less CAPE and 0-6-km shear.

The lower-shear Dumas Control simulation, using the 2257 UTC sounding, develops supercellular features within the first hour of the simulation and the mesocyclone frequently cycles throughout the next two hours, before ultimately dissipating (Fig. 14). At the time of BSS ( $t=$ $1.5 \mathrm{~h})$, the supercell is in the midst of cycling and no longer has an evident hook echo, despite large values of updraft helicity aloft. As the low-level shear increases in the higher-shear BSS simulation, the hook echo is more persistent and shows lower tendency toward splitting. Despite the countermeasures described in section $2 \mathrm{c}$, pronounced transverse rolls caused by Kelvin-Helmholtz instability (due to very large low-level shear) rapidly contaminate the simulation for the higher-shear BSS supercell after $3.5 \mathrm{~h}$ (not shown). As such, the analysis for this case will be focused on the first $3.5 \mathrm{~h}$ of the simulations.

The low-level mesocyclone response to the increase low-level shear is similar to the Goshen case (Fig. 15). Vertical vorticity values in the low-level mesocyclone increased by approximately $50 \%$. The higher-shear BSS supercell also readily produced intense near-surface 

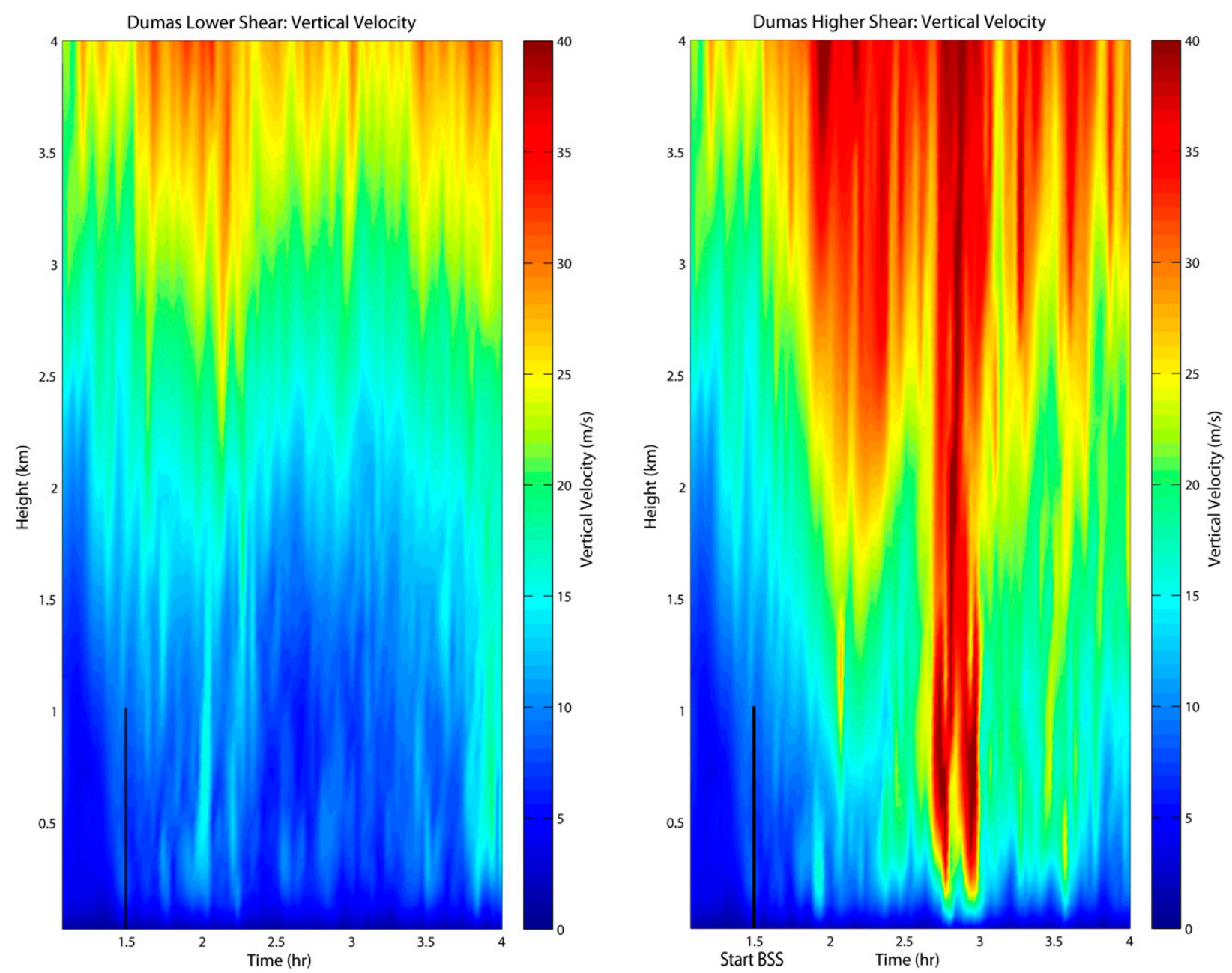

FIG. 16. As in Fig. 5, but for (left) the lower-shear Dumas Control simulation and (right) the higher-shear Dumas BSS simulation.

vorticity. The strongest vortex in the higher-shear BSS supercell strengthened to $0.25 \mathrm{~s}^{-1}$, more than 2.5 times as strong as any near-surface vortex in the lower-shear Control supercell. This again indicates that simply increasing the shear and storm-relative helicity during the EET can have profound impacts on the development of near-surface vorticity.

The higher-shear BSS supercell also has a stronger, deeper, and wider updraft than the lower-shear Control supercell (Figs. 16 and 17). The lowering of the updraft, evident in Fig. 16, occurs more rapidly than in the Goshen case, likely because the first Dumas BSS sounding at 0044 UTC incorporates almost as much modification as the final Goshen wind profile (due to the time gap between observed soundings in Fig. 2). The storm-averaged VPPGA shows immediate increases in the dynamic lifting due to the increased low-level shear and continues to increase until the supercell begins to weaken due to the growing Kelvin-Helmholtz instability mentioned previously (Fig. 18). Within $30 \mathrm{~min}$ after the completion of the BSS process, the acceleration in the low levels is several times stronger than the lowershear Control supercell (Fig. 18). The spatial extent of intense $0-1-\mathrm{km}$ dynamic lifting is particularly increased in the vicinity of hook echo (Fig. 19). The contribution of the nonlinear dynamic term to the low-level accelerations greatly exceeded the linear dynamic and buoyant terms (not shown). The dynamically enhanced low-level updraft in the higher-shear BSS supercell likely facilitated the development of a particularly intense nearsurface vortex, where the vorticity intensified to $0.25 \mathrm{~s}^{-1}$ and vertical velocities greater than $40 \mathrm{~m} \mathrm{~s}^{-1}$ extended below $300 \mathrm{~m}$ (Figs. 15 and 16). As in the Goshen case, outflow parcels with appreciable vorticity and identical buoyancy were more readily ingested into the parent updraft in the higher-shear supercell compared to its lower-shear counterpart (Fig. 20). Although we do not present them again for brevity, our calculations and 

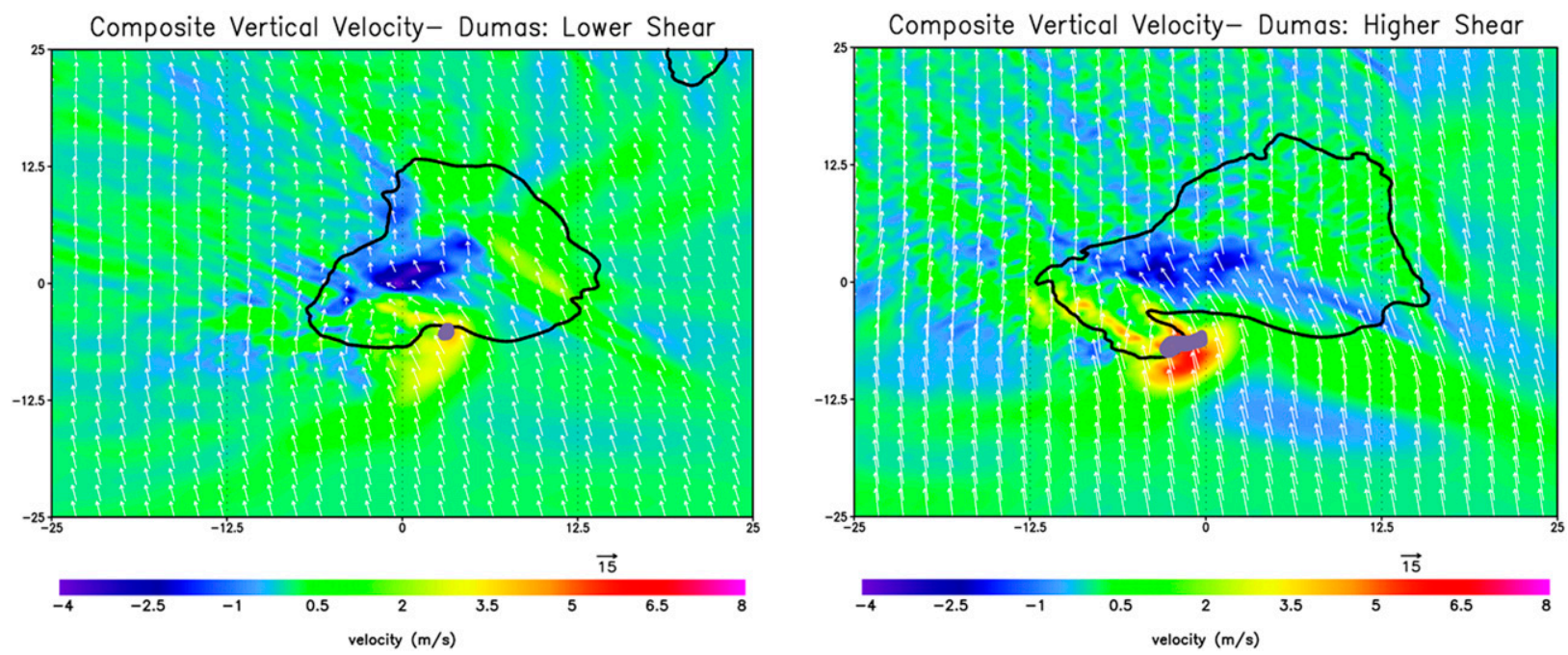

FIG. 17. As in Fig. 6, but for (left) the lower-shear Dumas Control simulation and (right) the higher-shear Dumas BSS simulation.

analyses for the Dumas case fully support the same explanations as were given for the Goshen case in section 3.

\section{Synthesis}

\section{a. Summary}

In this article, we investigated the role of increasing low-level shear on mature supercells during the EET using observed soundings from VORTEX2. Using BSS to gradually change the base state, supercells were simulated in environments with both moderate and high low-level shear for two different VORTEX2 cases. Previous work has indicated a relationship between strong low-level shear and the development of intense near-surface vorticity in supercells. Our simulations using slightly modified observed soundings show the following:
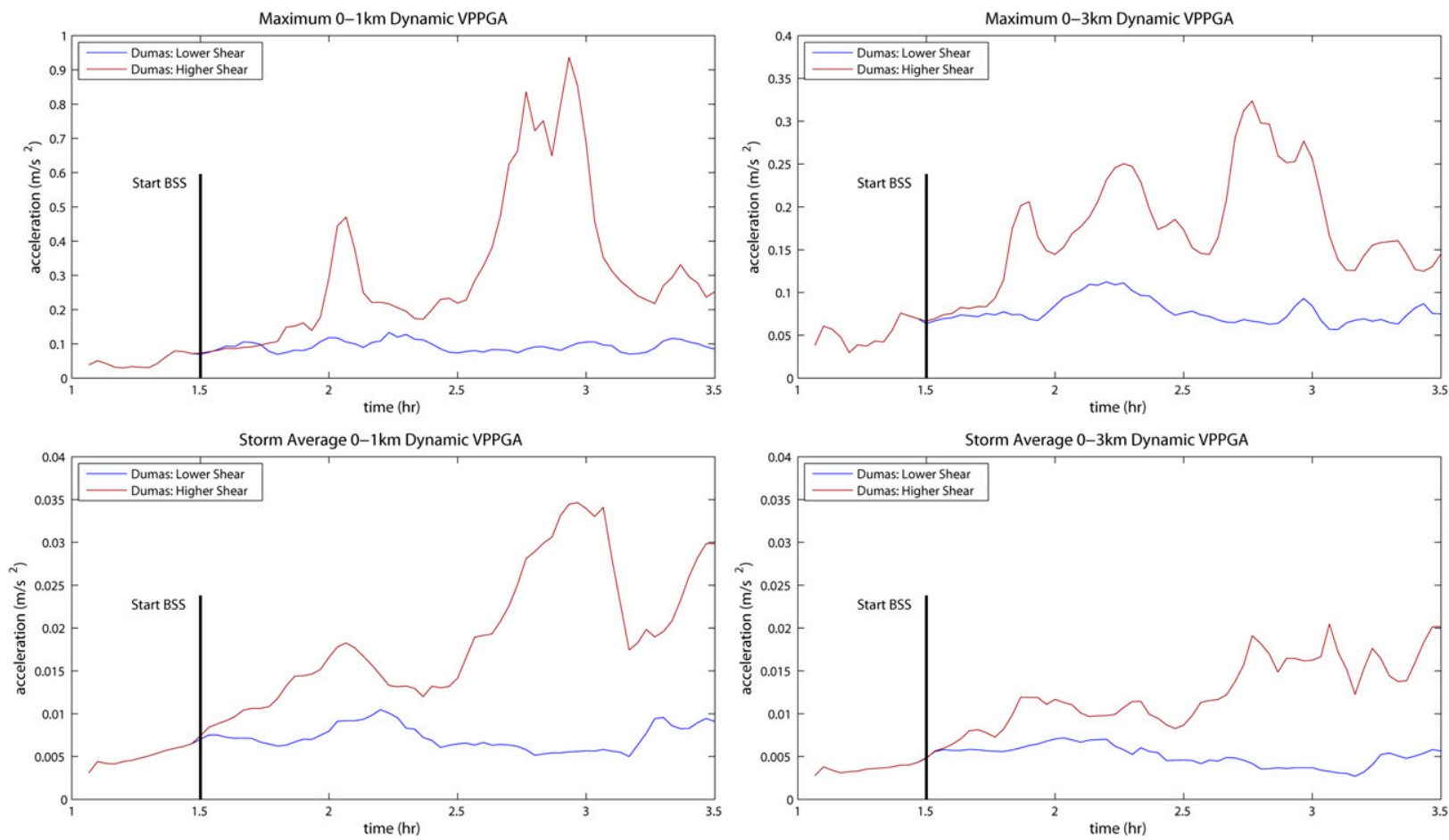

FIG. 18. As in Fig. 8, but for the lower-shear Dumas Control (blue) and the higher-shear Dumas BSS (red) supercells. 

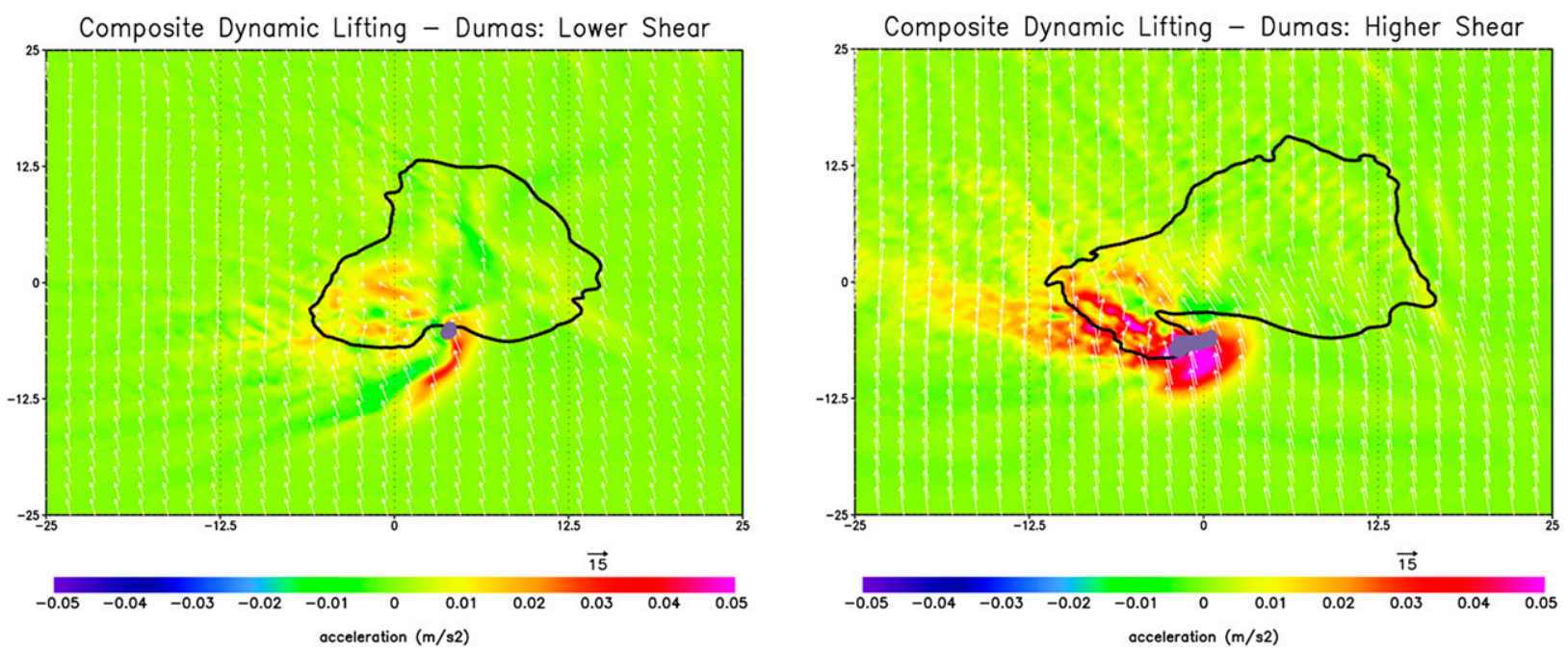

FIG. 19. As in Fig. 9, but for (left) the lower-shear Dumas Control simulation and (right) the higher-shear Dumas BSS simulation.

1) As low-level shear increases, the higher-shear supercells stayed more organized and displayed more coherent supercellular features (such as a hookecho, weak-echo regions, and increased updraft helicity) than the lower-shear supercells.

2) As low-level shear increases, the vertical vorticity throughout the depth of the storm was greatly enhanced in the higher-shear supercells, indicating that both the low-level and midlevel mesocyclones were enhanced by the increasing low-level shear. The updraft was more intense at low levels in the higher-shear supercells owing to the increased dynamic VPPGA associated with larger vertical vorticity within the supercell's updraft. This supports the conclusions from the idealized "pseudo-storm" study of Markowski and Richardson (2014).

\section{Bulk Characteristics for Outflow Trajectories: Dumas}
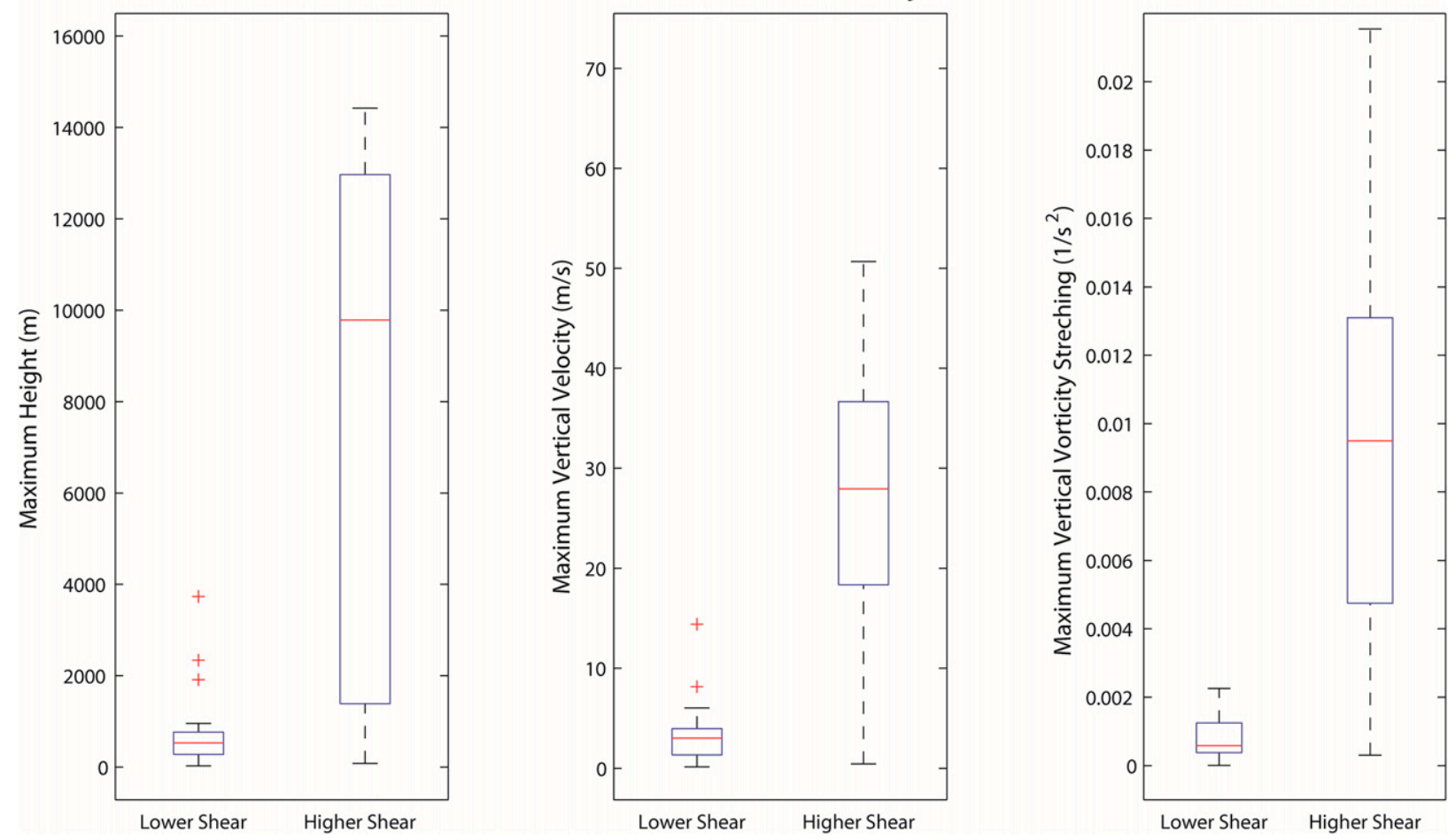

FIG. 20. As in Fig. 10, but for the lower-shear Dumas Control and the higher-shear Dumas BSS supercells. 
3) Both supercells produced appreciable near-surface vertical vorticity in their outflow, especially for the higher-shear supercells. Vorticity-rich outflow air was more readily ingested by the supercell's overlying updraft in the higher-shear supercells, despite similar buoyancy. The near-surface vortices were amplified by an increase in the dynamic component of the acceleration and vorticity stretching, which were the indirect result of the increased shear in the inflow environment.

4) When stabilization was introduced in order to emulate nocturnal cooling, the increases in low-level shear compensated for the decrease in low-level buoyancy. The intensity of the low-level updraft and near-surface rotation was only diminished after significant cooling had occurred in the higher-shear supercells.

These results suggest that low-level shear is operationally useful as a tornado forecasting parameter because the primary response of increasing low-level shear is to strengthen, widen, and lower the base of the mesocyclone, inducing stronger dynamic lifting that could forcibly lift outflow with appreciable vorticity. The trend of more intense nearsurface rotation with increasing low-level shear fits with our current understanding of tornadogenesis [as described in Davies-Jones (2015) and Markowski and Richardson (2014)]. Strengthened dynamic lifting provides a broad, persistent area where pretornadic surface vorticity can easily be concentrated and then subsequently stretched while participating in the parent updraft. Our findings also begin to explain the apparent linkage between the EET and tornado production. The early evening climatological maximum in tornado occurrence is probably not simply due to the fact that storms are often initiated in the mid- to late afternoon; EET-induced increases in lower-tropospheric vertical wind shear can play a large role in low-level vorticity and updraft intensification.

\section{b. Future work}

The details and specific sensitivities of the tornadogenesis process cannot be fully elucidated from this study, in part because of our grid spacing and in part because of some simplifications in our model configuration. The primary dynamical effects of shear upon lifting of outflow parcels via the pathway we have outlined should not, on principle, depend upon these numerical trade-offs. Nevertheless, in future work we plan to address the limitations imposed by the grid spacing, lack of surface drag, and our relatively simple microphysics parameterization. Naturally, there are other temporally varying factors that may influence the tornadogenesis process besides low-level shear. A larger matrix of simulations across a wide range of environments (e.g., with greater variations in low-level humidity and CAPE) would provide added context for the relative importance of changes in vertical wind shear. It is also notable that studies of nocturnal tornadoes [i.e., well after the early evening transition; e.g., Davies and Fischer (2009); Fischer and Davies (2009); Kis and Straka (2010); Mead and Thompson (2011)] imply a class of tornadic environments that are yet again distinct from those we have considered here.

An interesting ancillary finding from our work (as described briefly in section 2) is that it is rather difficult to carry out fully idealized storm simulations in environments with extremely strong vertical wind shear. Although not presented here, we attempted to simulate a third case from VORTEX2 (12 May 2010). The observed soundings showed temporal changes in low-level shear that were comparable to the Goshen and Dumas cases. However, the 12 May 2010 case possessed greater initial low-level shear than either of the final soundings in the Goshen and Dumas cases [Table 1 (bottom) and see Fig. S7 in the online supplemental material]. Simulations were attempted with several permutations of the observed soundings and model configurations (e.g., damping), but in every case KelvinHelmholtz instability led to growing perturbations that disrupted the simulated supercell. Given the apparent challenges presented by idealized simulations, it also may therefore be advantageous to exploit data assimilation techniques, which could be used to constrain the development of convection (i.e., so that the simulated environments and supercells are more similar to their real-life counterparts). The long-range goal of this effort is to advance our understanding of how environmental conditions influence within-storm processes, including tornadogenesis.

Acknowledgments. This research was supported by NSF Grant AGS-1156123. We thank Dr. George Bryan for his continual support of CM1 and Dr. Johannes Dahl for sharing his parcel interpolation/identification code. Drs. Casey Davenport (née Letkewicz) and Adam French are acknowledged for developing and providing their BSS modifications to CM1. We thank Dr. Matthew Bunkers and two anonymous reviewers for providing a thorough review that greatly improved this manuscript. The first author's committee members, Drs. Gary Lackmann and Sandra Yuter, provided helpful feedback of an earlier version of this manuscript. Finally, we appreciate the support of current/past members of the NCSU Convective Storms Group, including Jason Davis, Jessica King, Chris MacIntosh, and Keith Sherburn.

\section{REFERENCES}

Adlerman, E. J., K. K. Droegemeier, and R. P. Davies-Jones, 1999: A numerical simulation of cyclic mesocyclogenesis. J. Atmos. Sci., 56, 2045-2069, doi:10.1175/1520-0469(1999)056<2045: ANSOCM $>2.0 . \mathrm{CO} ; 2$. 
Atkins, N. T., A. McGee, R. Ducharme, R. M. Wakimoto, and J. Wurman, 2012: The LaGrange Tornado during VORTEX2. Part II: Photogrammetric analysis of the tornado combined with dual-Doppler radar data. Mon. Wea. Rev., 140, 29392958, doi:10.1175/MWR-D-11-00285.1.

Acevedo, O. C., and D. R. Fitzjarrald, 2001: The early evening surface-layer transition: Temporal and spatial variability. J. Atmos. Sci, 58, 2650-2667, doi:10.1175/1520-0469(2001)058<2650: TEESLT>2.0.CO;2.

Blackadar, A. K., 1957: Boundary layer wind maxima and their significance for the growth of nocturnal inversions. Bull. Amer. Meteor. Soc., 38, 282-290.

Bonner, W. D., 1968: Climatology of the low level jet. Mon. Wea. Rev., 96, 833-850, doi:10.1175/1520-0493(1968)096<0833: COTLLJ $>2.0 . \mathrm{CO} ; 2$

Brooks, H. E., J. W. Lee, and J. P. Craven, 2003: The spatial distribution of severe thunderstorm and tornado environments from global reanalysis data. Atmos. Res., 67-68, 73-94, doi:10.1016/ S0169-8095(03)00045-0.

Bryan, G. H., and J. M. Fritsch, 2002: A benchmark simulation for moist nonhydrostatic numerical models. Mon. Wea. Rev., 130, 2917-2928, doi:10.1175/1520-0493(2002)130<2917: ABSFMN $>2.0 . \mathrm{CO} ; 2$.

Bunkers, M. J., B. A. Klimowski, J. W. Zeitler, R. L. Thompson, and M. L. Weisman, 2000: Predicting supercell motion using a new hodograph technique. Wea. Forecasting, 15, 61-79, doi:10.1175/1520-0434(2000)015<0061:PSMUAN>2.0.CO;2.

Dahl, J. M. L., M. D. Parker, and L. J. Wicker, 2014: Imported and storm-generated near-ground vertical vorticity in a simulated supercell. J. Atmos. Sci., 71, 3027-3051, doi:10.1175/ JAS-D-13-0123.1.

Davies, J. M., and A. Fischer, 2009: Environmental characteristics associated with nighttime tornadoes. Electron. J. Oper. Meteor., 10 (3), 1-29. [Available online at http://www.nwas.org/ej/ 2009-EJ3/.]

Davies-Jones, R. P., 2015: A review of supercell and tornado dynamics. Atmos. Res., doi:10.1016/j.atmosres.2014.04.007, in press.

—_ and H. E. Brooks, 1993: Mesocyclogenesis from a theoretical perspective. The Tornado: Its Structure, Dynamics, Prediction, and Hazards, Geophys. Monogr., Vol. 79, Amer. Geophys. Union, 105-114.

_ nadic storms. Severe Convective Storms, Meteor. Monogr., No. 28, Amer. Meteor. Soc., 167-222, doi:10.1175/0065-9401-28.50.167.

Doswell, C. A., 1980: Synoptic-scale environments associated with High Plains severe thunderstorms. Bull. Amer. Meteor. Soc., 61, 1388-1400, doi:10.1175/1520-0477(1980)061<1388: SSEAWH $>2.0 . \mathrm{CO} ; 2$.

Fischer, A., and J. M. Davies, 2009: Significant nighttime tornadoes in the plains associated with relatively stable low-level conditions. Electron. J. Oper. Meteor., 10 (4), 1-33. [Available online at http://www.nwas.org/ej/2009-EJ4/.]

Frame, J., and P. M. Markowski, 2010: Numerical simulations of radiative cooling beneath the anvils of supercell thunderstorms. Mon. Wea. Rev., 138, 3024-3047, doi:10.1175/ 2010MWR3177.1.

— , and —, 2013: Dynamical influences of anvil shading on simulated supercell thunderstorms. Mon. Wea. Rev., 141, 2802-2820, doi:10.1175/MWR-D-12-00146.1.

French, A. J., and M. D. Parker, 2010: The response of simulated nocturnal convective systems to a developing low-level jet. J. Atmos. Sci., 67, 3384-3408, doi:10.1175/2010JAS3329.1.
French, M. M., H. B. Bluestein, D. C. Dowell, L. J. Wicker, M. R. Kramar, and A. L. Pazmany, 2008: High-resolution, mobile Doppler radar observations of cyclic mesocyclogenesis in a supercell. Mon. Wea. Rev., 136, 4997-5016, doi:10.1175/ 2008MWR2407.1.

Fritsch, J. M., J. D. Murphy, and J. S. Kain, 1994: Warm core vortex amplification over land. J. Atmos. Sci., 51, 1780-1807, doi:10.1175/ 1520-0469(1994)051<1780:WCVAOL $>2.0 . C O ; 2$.

Kain, J. S., and Coauthors, 2008: Some practical considerations regarding horizontal resolution in the first generation of operational convection-allowing NWP. Wea. Forecasting, 23, 931-952, doi:10.1175/WAF2007106.1.

Kis, A. K., and J. M. Straka, 2010: Nocturnal tornado climatology. Wea. Forecasting, 25, 545-561, doi:10.1175/ 2009WAF2222294.1.

Klemp, J. B., and R. B. Wilhelmson, 1978: The simulation of three-dimensional convective storm dynamics. J. Atmos. Sci., 35, 1070-1096, doi:10.1175/1520-0469(1978)035<1070: TSOTDC $>2.0 . \mathrm{CO} ; 2$.

Kosiba, K., J. Wurman, Y. Richardson, P. Markowski, P. Robinson, and J. Marquis, 2013: Genesis of the Goshen County, Wyoming, tornado on 5 June 2009 during VORTEX2. Mon. Wea. Rev., 141, 1157-1181, doi:10.1175/ MWR-D-12-00056.1.

Lapworth, A., 2003: Factors determining the decrease in surface wind speed following the evening transition. Quart. J. Roy. Meteor. Soc., 129, 1945-1968, doi:10.1256/qj.02.163.

Letkewicz, C. E., A. J. French, and M. D. Parker, 2013: Base-state substitution: An idealized modeling technique for approximating environmental variability. Mon. Wea. Rev., 141, 30623086, doi:10.1175/MWR-D-12-00200.1.

Lin, Y.-L., R. D. Farley, and H. D. Orville, 1983: Bulk parameterization of the snow field in a cloud model. J. Climate Appl. Meteor., 22, 1065-1089, doi:10.1175/1520-0450(1983)022<1065: BPOTSF $>2.0 . \mathrm{CO} ; 2$.

Loftus, A. M., D. B. Weber, and C. A. Doswell, 2008: Parameterized mesoscale forcing mechanisms for initiating numerically simulated isolated multicellular convection. Mon. Wea. Rev., 136, 2408-2421, doi:10.1175/2007MWR2133.1.

Maddox, R. A., 1993: Diurnal low-level wind oscillation and storm-relative helicity. The Tornado: Its Structure, Dynamics, Prediction, and Hazards, Geophys. Monogr., Vol. 79, Amer. Geophys. Union, 591-598.

Mansell, E. R., C. L. Ziegler, and E. C. Bruning, 2010: Simulated electrification of a small thunderstorm with two-moment bulk microphysics. J. Atmos. Sci., 67, 171-194, doi:10.1175/ 2009JAS2965.1.

Markowski, P. M., and Y. P. Richardson, 2010: Mesoscale Meteorology in Midlatitudes. Wiley-Blackwell, $407 \mathrm{pp}$.

_, and _ 2014: The influence of environmental low-level shear and cold pools on tornadogenesis: Insights from idealized simulations. J. Atmos. Sci., 71, 243-275, doi:10.1175/ JAS-D-13-0159.1.

J. M. Straka, E. N. Rasmussen, and D. C. Dowell, 1998a: Observations of low-level baroclinity generated by anvil shadows. Mon. Wea. Rev., 126, 2942-2958, doi:10.1175/15200493(1998)126<2942:OOLLBG > 2.0.CO;2.

,$--\longrightarrow$, and D. O. Blanchard, 1998b: Variability of storm-relative helicity during VORTEX. Mon. Wea. Rev., 126, 2959-2971, doi:10.1175/1520-0493(1998)126<2959: VOSRHD $>2.0 . \mathrm{CO} ; 2$

,-- , and - 2002: Direct surface thermodynamic observations within the rear-flank downdrafts of nontornadic and 
tornadic supercells. Mon. Wea. Rev., 130, 1692-1721, doi:10.1175/ 1520-0493(2002)130<1692:DSTOWT>2.0.CO;2.

, C. Hannon, J. Frame, E. Lancaster, A. Pietrycha, R. Edwards, and R. L. Thompson, 2003: Characteristics of vertical wind profiles near supercells obtained from the Rapid Update Cycle. Wea. Forecasting, 18, 1262-1272, doi:10.1175/ 1520-0434(2003)018<1262:COVWPN >2.0.CO;2.

- J. M. Straka, E. N. Rasmussen, R. P. Davies-Jones, Y. Richardson, and R. J. Trapp, 2008: Vortex lines within low-level mesocyclones obtained from pseudo-dual-Doppler radar observations. Mon. Wea. Rev., 136, 3513-3535, doi:10.1175/ 2008MWR2315.1.

, Y. P. Richardson, M. Majcen, J. Marquis, and J. Wurman, 2011: Characteristics of the wind field in three nontornadic low-level mesocyclones observed by the Doppler On Wheels radars. Electron. J. Severe Storms Meteor., 6 (3). [Available online at http://www.ejssm.org/ojs/index.php/ejssm/article/ viewArticle/75.]

_ County, Wyoming, supercell of 5 June 2009 intercepted by VORTEX2. Part I: Evolution of kinematic and surface thermodynamic fields. Mon. Wea. Rev., 140, 2887-2915, doi:10.1175/MWR-D-11-00336.1.

— , and Coauthors, 2012b: The pretornadic phase of the Goshen County, Wyoming, supercell of 5 June 2009 intercepted by VORTEX2. Part II: Intensification of low-level rotation. Mon. Wea. Rev., 140, 2916-2938, doi:10.1175/ MWR-D-11-00337.1.

Marquis, J., Y. P. Richardson, P. M. Markowski, D. Dowell, and J. Wurman, 2012: Tornado maintenance investigated with high-resolution dual-Doppler and EnKF analysis. Mon. Wea. Rev., 140, 3-27, doi:10.1175/MWR-D-11-00025.1.

,,,--- J. Wurman, K. Kosiba, P. Robinson, and G. Romine, 2014: An investigation of the Goshen County, Wyoming, tornadic supercell of 5 June 2009 using EnKF assimilation of mobile mesonet and radar observations collected during VORTEX2. Part I: Experiment design and verification of the EnKF analyses. Mon. Wea. Rev., 142, 530 554, doi:10.1175/MWR-D-13-00007.1.

McCaul, E. W., and M. L. Weisman, 1996: Simulations of shallow supercell storms in landfalling hurricane environments. Mon. Wea. Rev., 124, 408-429, doi:10.1175/1520-0493(1996)124<0408: SOSSSI $>2.0 . \mathrm{CO} ; 2$.

Mead, C. M., and R. L. Thompson, 2011: Environmental characteristics associated with nocturnal significant-tornado events in the Great Plains. Electron. J. Severe Storms Meteor., 6 (6). [Available online at http://www.ejssm.org/ojs/index.php/ ejssm/article/viewArticle/84.]

Morrison, H., J. A. Curry, and V. I. Khvorostyanov, 2005: A new double-moment microphysics parameterization for application in cloud and climate models. Part I: Description. J. Atmos. Sci., 62, 1665-1677, doi:10.1175/JAS3446.1.

Nieuwstadt, F. T. M., 1985: A model for the stationary, stable boundary layer. Turbulence and Diffusion in Stable Environments, J. C. R. Hunt, Ed., Oxford University Press, 149-179.

Nowotarski, C. J., P. M. Markowski, and Y. P. Richardson, 2011: The characteristics of numerically simulated supercell storms situated over statically stable boundary layers. Mon. Wea. Rev., 139, 3139-3162, doi:10.1175/MWR-D-10-05087.1.

,,-- , and G. H. Bryan, 2015: Supercell low-level mesocyclones in simulations with a sheared convective boundary layer. Mon. Wea. Rev., 143, 272-297, doi:10.1175/ MWR-D-14-00151.1.
Parker, M. D., 2014: Composite VORTEX2 supercell environments from near-storm soundings. Mon. Wea. Rev., 142, 508529, doi:10.1175/MWR-D-13-00167.1.

Richardson, Y. P., K. K. Droegemeier, and R. P. Davies-Jones, 2007: The influence of horizontal environmental variability on numerically simulated convective storms. Part I: Variations in vertical shear. Mon. Wea. Rev., 135, 3429-3455, doi:10.1175/ MWR3463.1.

Rotunno, R., and J. B. Klemp, 1982: The influence of the shearinduced pressure gradient on thunderstorm motion. Mon. Wea. Rev., 110, 136-151, doi:10.1175/1520-0493(1982)110<0136: TIOTSI $>2.0 . \mathrm{CO} ; 2$.

Schenkman, A. D., M. Xue, and M. Hu, 2014: Tornadogenesis in a high-resolution simulation of the 8 May 2003 Oklahoma City supercell. J. Atmos. Sci., 71, 130-154, doi:10.1175/ JAS-D-13-073.1.

Shapiro, A., and E. Fedorovich, 2010: Analytical description of a nocturnal low-level jet. Quart. J. Roy. Meteor. Soc., 136, 1255-1262, doi:10.1002/qj.628.

Skinner, P., C. Weiss, M. French, H. Bluestein, P. Markowski, and Y. Richardson, 2014: VORTEX2 observations of a low-level mesocyclone with multiple internal real-flank downdraft momentum surges in the 18 May 2010 Dumas, Texas, supercell. Mon. Wea. Rev., 142, 2935-2960, doi:10.1175/ MWR-D-13-00240.1.

Song, J., K. Liao, R. L. Coulter, and B. M. Lesht, 2005: Climatology of the low-level jet at the southern Great Plains Atmospheric Boundary Layer Experiment site. J. Appl. Meteor., 44, 15931606, doi:10.1175/JAM2294.1.

Stull, R. B., 1988: An Introduction to Boundary Layer Meteorology. Kluwer Academic, 666 pp.

Tao, W.-K., and J. Simpson, 1993: The Goddard Cumulus Ensemble Model. Part I: Model description. Terr. Atmos. Oceanic Sci., 4, 19-54.

Thompson, G., P. R. Field, R. M. Rasmussen, and W. D. Hall, 2008: Explicit forecasts of winter precipitation using an improved bulk microphysics scheme. Part II: Implementation of a new snow parameterization. Mon. Wea. Rev., 136, 50955115, doi:10.1175/2008MWR2387.1.

Thompson, R. L., R. Edwards, J. A. Hart, K. L. Elmore, and P. Markowski, 2003: Close proximity soundings within supercell environments obtained from the rapid update cycle. Wea. Forecasting, 18, 1243-1261, doi:10.1175/1520-0434(2003)018<1243: CPSWSE $>2.0 . \mathrm{CO} ; 2$.

— C. M. Mead, and R. Edwards, 2007: Effective storm-relative helicity and bulk shear in supercell thunderstorm environments. Wea. Forecasting, 22, 102-115, doi:10.1175/WAF969.1.

Trapp, R. J., 1999: Observations of nontornadic low-level mesocyclones and attendant tornadogenesis failure during VORTEX. Mon. Wea. Rev., 127, 1693-1705, doi:10.1175/ 1520-0493(1999)127<1693:OONLLM>2.0.CO;2.

_ G. J. Stumpf, and K. L. Manross, 2005: A reassessment of the precentage of tornadic mesocyclones. Wea. Forecasting, 20, 680-687, doi:10.1175/WAF864.1.

Van de Wiel, B. J. H., A. F. Moene, G. J. Steeneveld, P. Baas, F. C. Bosveld, and A. M. Holtslag, 2010: A conceptual view on inertial oscillations and nocturnal low-level jets. J. Atmos. Sci., 67, 2679-2689, doi:10.1175/2010JAS3289.1.

Wakimoto, R. M., N. T. Atkins, and J. Wurman, 2011: The LaGrange Tornado during VORTEX2. Part I: Photogrammetric analysis of the tornado combined with single-Doppler radar data. Mon. Wea. Rev., 139, 2233-2258, doi:10.1175/ 2010MWR3568.1. 
, P. Stauffer, W. Lee, N. T. Atkins, and J. Wurman, 2012: Finescale structure of the LaGrange, Wyoming, tornado during VORTEX2: GBVTD and photogrammetric analyses. Mon. Wea. Rev., 140, 3397-3418, doi:10.1175/MWR-D-12-00036.1.

Wilhelmson, R. B., and Y. Ogura, 1972: The pressure perturbation and the numerical modeling of a cloud. J. Atmos. Sci., 29, 1295-1307, doi:10.1175/1520-0469(1972)029<1295: TPPATN $>2.0 . \mathrm{CO} ; 2$.
— , and L. J. Wicker, 2001: Numerical modeling of severe local storms. Severe Convective Storms, Meteor. Monogr., No. 28, Amer. Meteor. Soc., 123-166, doi:10.1175/0065-9401-28.50.123.

Wurman, J., D. Dowell, Y. Richardson, P. Markowski, E. Rasmussen, D. Burgess, L. Wicker, and H. B. Bluestein, 2012: The Second Verification of the Origins of Rotation in Tornadoes Experiment: VORTEX2. Bull. Amer. Meteor. Soc., 93, 11471170, doi:10.1175/BAMS-D-11-00010.1. 